\title{
A New Four-dimensional Ratcheting Boundary: Derivation and Numerical Validation
}

\author{
Jun Shen ${ }^{1}$, Haofeng Chen²*, Yinghua Liu
}

1. School of Aerospace Engineering, Tsinghua University, Beijing 100084, P.R. China

2. Department of Mechanical \& Aerospace Engineering, University of Strathclyde, Glasgow

G1 1XJ, UK

\section{Abstract}

A new four-dimensional ratcheting boundary is derived analytically for the first time considering the interaction among four types of stresses: constant mechanical membrane stress, mechanical bending stress, cyclic thermal membrane stress, and thermal bending stress. A uniaxial beam model is used to derive the closed-form ratcheting boundary for these combined cyclic and constant loadings. The Tresca yield condition and elastic-perfectly plastic behavior are assumed. A novel two-plane FE model is proposed for numerical validation and the results predicted by analytical solution agree very well with that obtained by two-plane FE model. The solution of the classical Bree problem is the one of special cases when this new four-dimensional ratcheting boundary is reduced into two-dimensional style. The relationship between the threedimensional ratcheting boundary adopted by the newly implemented ASME VIII 2 Pressure Vessel Code and the proposed four-dimensional ratcheting boundary is also discussed.

\section{Key words}

Ratchet boundary; Shakedown; Two-plane model; Plastic FEA; Noncyclic method

\section{Nomenclature}

\begin{tabular}{l}
\cline { 2 - 2 } Description \\
\hline${ }^{2}$ Author to whom correspondence should be addressed; E-mail:haofeng.chen@strath.ac.uk
\end{tabular}




\begin{tabular}{ll}
\hline$\sigma_{y}$ & Cyclic Yield Stress or Initial Yield \\
$\sigma_{\mathrm{sm}}$ & Stress \\
$\sigma_{\mathrm{sb}}$ & Thermal Membrane Stress \\
$\sigma_{\mathrm{pb}}$ & Mechanical Bending Stress \\
$\sigma_{\mathrm{pm}}$ & Mechanical Membrane Stress \\
$\Delta \sigma_{\mathrm{sm}}$ & Thermal Membrane Stress Range \\
$\Delta \sigma_{\mathrm{sb}}$ & Thermal Bending Stress Range \\
$\sigma_{y}^{\prime}(y)$ & Remaining Yield Stress Distribution \\
$h$ & Height of Rectangular Beam \\
$w$ & Width of Rectangular Beam \\
$F_{p}$ & Axial Load Corresponding \\
$M_{p}$ & To Ratchet Boundary \\
\hline
\end{tabular}

\section{Introduction}

Structures and components used in chemical, petroleum, military, and nuclear power plants are usually subject to a combination of sustained and cyclic loadings, such as internal pressure and high temperature gradient, tension and torsion, bending and torsion, and so on[1]. When the combination of the applied cyclic and constant load levels exceeds the ratchet limit, the plastic deformation may be accumulated during each load cycle, leading to an incremental plastic collapse, i.e. ratcheting [2].

For the design of components, ratcheting is usually not an acceptable behavior in engineering. So a considerable amount of theoretical and numerical research on ratcheting analysis under complex stress state was carried out over the past few decades. One of the most famous and classic analytical solution was given by Bree (1967) [3, 4]. The two-dimensional Bree diagram shows the ratcheting boundary of a cylindrical shell subjected to the combination of internal pressure and cycled linear distributed temperature across the shell wall. The constant mechanical membrane stress and cyclic thermal stress caused by above two types of loads are represented by abscissa and ordinate respectively in this diagram which is adopted by ASME VIII-2 Code 2011 [5] and previous versions. However, it is still difficult to obtain the analytical solutions of ratcheting limits for complex stress state and more universal constraints by theoretical derivation.

Until now, many investigations [6-9] about ratcheting boundary for cylindrical shell have only considered a few stress parameters of these four stresses: constant mechanical membrane stress, mechanical bending stress, cyclic thermal membrane stress, and thermal bending stress. W.Bradford et al. [6,7] derived shakedown and ratcheting behavior for uniaxial primary membrane and thermal bending stresses which was compared with the results of the linear matching method (LMM) $[10,11]$ and excellent agreement was found. The proposed ratcheting 
evaluation method $[8,9]$ considering three stresses of the four was already adopted by ASME VIII-2 Code 2013 [12] and later versions. Nevertheless, the unified ratcheting analytical solution for these four types of stresses is still unavailable due to complex effects of interaction on each other, as well as the difficulty of building the numerical model for validation.

In the present paper, analytical solutions of ratchet boundary for constant mechanical membrane stress and mechanical bending stress, cyclic thermal membrane stress and thermal bending stress were derived based on the developed noncyclic method. In the second part, the nomenclatures used were presented. In the third part, the noncyclic method is introduced. In the fourth and fifth parts, detailed analytical derivation and related discussion were given out. In the sixth part, a novel two-plane FE model is built to verify the current analytical solutions. Finally, conclusions are summarized.

\section{Outline of Noncyclic method}

Noncyclic analysis methods that use Melan's theorem have been proposed for elastic shakedown analysis and plastic shakedown analysis by W. Reinhardt [13]. The outline of the method is decomposing a loading history into fully reversed cyclic and constant loadings, and then analyzing them separately. For an actual structure under loading history, the detailed analyses for the ratchet boundary using the noncyclic method can involve the following steps [13]:

(1)Decompose the loading into constant and fully reversed cyclic loadings.

(2)Create the finite element model of the structure only subjected to the cyclic loading, and perform elastic-perfectly plastic analyses with the initial yield strength $\sigma_{y}$.

(3)For each location (element), the remaining yield strength $\sigma_{y}^{\prime}(y)$ can be obtained by subtracting the von Mises equivalent stress from the cyclic yield strength $\sigma_{y}$. Then the remaining yield strength $\sigma_{y}^{\prime}(y)$ is used to the structure as the new yield strength.

(4)Apply the constant load only and perform a limit analysis for the structure with remaining yield strength $\sigma_{y}^{\prime}(y)$, then the limit load corresponding to the plastic collapse of the structure is the lower bound of the allowable constant load for the ratchet boundary.

Several analytical derivations of the ratchet boundary based on the noncyclic method can be found in previous literatures. For example, the Bree problem and inverse Bree problem have been demonstrated in Ref [13], and the expression of ratchet boundary for the rectangular beam subjected to steady mechanical membrane, cyclic thermal membrane and cyclic thermal bending stress simultaneously is derived in Ref [8], and the ratchet limit solution of a beam with arbitrary cross section is obtained analytically in Ref [9] for the case that the cyclic thermal bending and the combination of a steady mechanical membrane and mechanical bending load act simultaneously. However, only three types of stresses or less were considered at the same time in the analytic solutions mentioned above.

\section{Analytical Derivation}

\subsection{General cases}

In this section, the noncyclic method is extended to derive the ratchet boundary for an elastic-perfectly plastic beam subjected to cyclic thermal bending stress, cyclic thermal membrane stress and the combination of a steady mechanical membrane stress and mechanical bending stress. 
For the sake of the noncyclic method, the thermal bending and membrane stresses are assumed to cycle simultaneously and in-phase, and vary around a zero mean stress [2].

In noncyclic method, the cyclic thermal loads are applied first, then the thermal stress amplitude including thermal bending stress and thermal membrane stress at each location is determined. Due to uniaxial stress field in a beam, the von Mises equivalent stress is equal to the absolute value of uniaxial stress. Thus, the remaining yield stress distribution $\sigma_{y}^{\prime}(y)$ to support subsequent mechanical load is obtained by subtracting this amplitude from the initial cyclic yield stress $\sigma_{y}$ at each location. It should be noted that for the case where the thermal stress amplitude is beyond the cyclic yield stress $\sigma_{y}$ at special region, such as the outer fibers of the beam, the special region will have zero remaining yield stress when the subsequent load components are applied, i.e. $\sigma_{y}^{\prime}=0$ in this region, and will thus not contribute to the carrying capacity of the beam for the subsequent mechanical stress. In the next step, the beam with the remaining yield stress $\sigma_{y}^{\prime}(y)$ is subjected to the subsequent steady mechanical loads. Through a limit analysis under all the steady mechanical loads for the beam with the remaining yield stress $\sigma_{y}^{\prime}(y)$, the mechanical membrane and bending stresses corresponding to the ratchet boundary are determined.

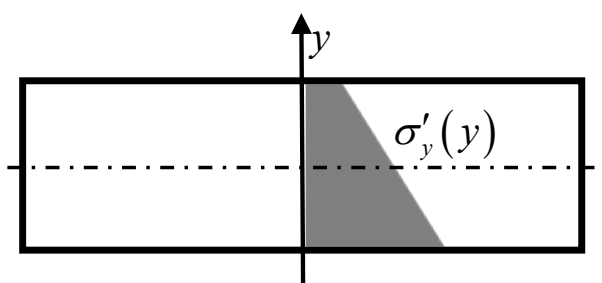

Figure 1. The assuming distributions of the remaining yield stress $\sigma_{y}^{\prime}(y)$

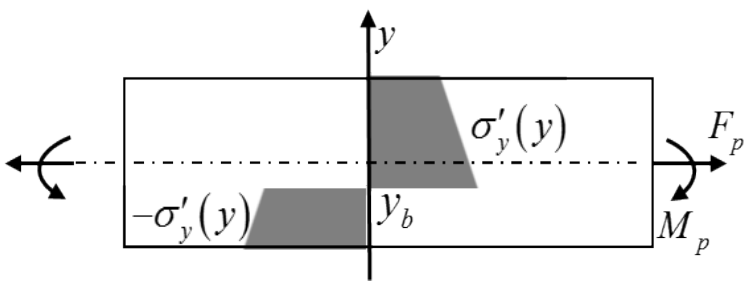

Figure 2. The assuming uniaxial stress distributions in limit state under mechanical axial load and mechanical bending load

In order to perform uniformly the limit analyses for the beam with the remaining yield stress $\sigma_{y}^{\prime}(y)$, the assumption is established that the remaining yield stress distribution $\sigma_{y}^{\prime}(y)$ is shown in Figure 1 and the uniaxial stress distributions in limit state under mechanical axial load and mechanical bending load is depicted in Figure 2. The height coordinate of the beam is $y$, and the origin of the coordinate is the intersection of the neutral axial and y-direction. The cross section is assumed to be a rectangle with the height $h$ and the width $w$. Then the limit analysis is performed in the following by establishing the equilibrium equation through the thickness from Figure 2:

$$
F_{p}=\int_{y_{b}}^{\frac{h}{2}} \sigma_{y}^{\prime}(y) w d y-\int_{-\frac{h}{2}}^{y_{b}} \sigma_{y}^{\prime}(y) w d y
$$




$$
M_{p}=\int_{y_{b}}^{\frac{h}{2}} y \sigma_{y}^{\prime}(y) w d y-\int_{-\frac{h}{2}}^{y_{b}} y \sigma_{y}^{\prime}(y) w d y
$$

where $F_{p}, M_{p}$ and $y_{b}$ are the limit mechanical axial force, the limit mechanical bending moment and the boundary coordinate between the positive stress and negative stress respectively.

On the other hand, for a rectangular beam, the following expressions for $F_{p}, M_{p}$ are valid[9]:

$$
\begin{gathered}
F_{p}=w h \sigma_{\mathrm{pm}} \\
M_{p}=\frac{w h^{2} \sigma_{\mathrm{pb}}}{6}
\end{gathered}
$$

where $\sigma_{\mathrm{pm}}$ and $\sigma_{\mathrm{pb}}$ are separately the steady mechanical membrane and bending stress. Through combining Eq.(1) (4), $\sigma_{\mathrm{pm}}$ and $\sigma_{\mathrm{pb}}$ can be obtained and expressed as follows:

$$
\begin{gathered}
\sigma_{\mathrm{pm}}=\frac{1}{h}\left(\int_{y_{b}}^{\frac{h}{2}} \sigma_{y}^{\prime}(y) d y-\int_{-\frac{h}{2}}^{y_{b}} \sigma_{y}^{\prime}(y) d y\right) \\
\sigma_{\mathrm{pb}}=\frac{6}{h^{2}}\left(\int_{y_{b}}^{\frac{h}{2}} y \sigma_{y}^{\prime}(y) d y-\int_{-\frac{h}{2}}^{y_{b}} y \sigma_{y}^{\prime}(y) d y\right)
\end{gathered}
$$

It should be noted that Eqs. (1), (2), (5) and (6) are the general expressions of the ratchet boundary for undetermined remaining yield stress distribution. Therefore, if the remaining yield stress distribution is known, then $\sigma_{\mathrm{pm}}$ and $\sigma_{\mathrm{pb}}$ for the ratchet boundary can be obtained by the Eq.(5) and (6).

For the case where the thermal membrane and thermal bending stresses act simultaneously, according to whether the thermal stress amplitude exceeds the cyclic yield stress or not, there are five characteristic distributions of the thermal cyclic stress amplitude[8], as shown in Figure 3. 

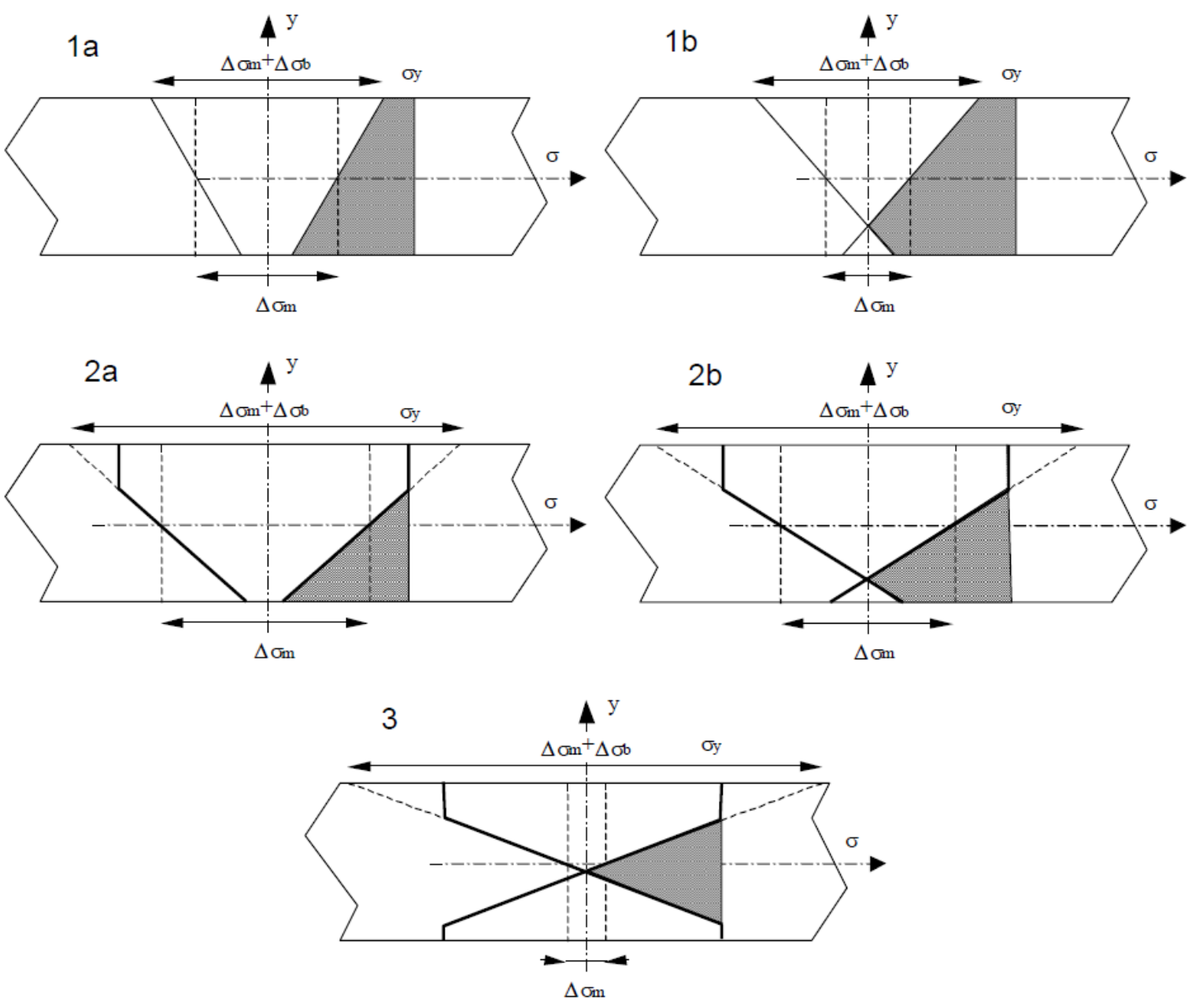

Figure 3. Characteristic distributions of the thermal cyclic stress amplitude (the shaded areas represent the remaining yield stress $\left.\sigma_{y}^{\prime}(y)\right)[8]$

It is clear from Figure 3 that the five characteristic distributions of the thermal cyclic stress amplitude can be separated into three typical cases: the first case denoted ' 1 ' (i.e. case 1a and 1b) corresponds to the elastic cycling situation when the cyclic thermal stress is low enough not to cause yielding through all the cross section. The second case denoted ' 2 ' (i.e. case $2 \mathrm{a}$ and $2 b)$ represents that the cyclic thermal stress at the outer fibres from one surface reaches the initial cyclic yield strength $\sigma_{y}$ and the third case denoted ' 3 ' expresses that the cyclic thermal stress causes yielding of the outer fibres from both surfaces. Moreover, the difference between cases $1 \mathrm{a}, 2 \mathrm{a}$ and cases $1 \mathrm{~b}, 2 \mathrm{~b}, 3$ is found that the thermal bending contribution to the total cyclic thermal stress is larger in cases $1 \mathrm{a}$ and $2 \mathrm{a}$ whereas the thermal membrane contribution is larger in cases $1 b, 2 b$, and 3 . Therefore, different thermal stress distribution through the cross section leads to five characteristic expressions of the remaining yield stress $\sigma_{y}^{\prime}(y)$, which is expressed as follows:

Case 1a corresponding the condition $\Delta \sigma_{\mathrm{sm}}+\Delta \sigma_{\mathrm{sb}} \leq 2 \sigma_{\mathrm{y}}$ and: $\Delta \sigma_{\mathrm{sm}} \geq \Delta \sigma_{\mathrm{sb}}$

$$
\sigma_{y}^{\prime}(y)=\sigma_{\mathrm{y}}-\frac{\Delta \sigma_{\mathrm{sm}}}{2}-\frac{\Delta \sigma_{\mathrm{sb}}}{h} y
$$


Case $1 \mathrm{~b}$ corresponding the condition $\Delta \sigma_{\mathrm{sm}}+\Delta \sigma_{\mathrm{sb}} \leq 2 \sigma_{\mathrm{y}}$ and $\Delta \sigma_{\mathrm{sm}} \leq \Delta \sigma_{\mathrm{sb}}$ :

$$
\sigma_{y}^{\prime}(y)= \begin{cases}\sigma_{\mathrm{y}}+\frac{\Delta \sigma_{\mathrm{sm}}}{2}+\frac{\Delta \sigma_{\mathrm{sb}}}{h} y, & y \in\left[-\frac{h}{2},-\frac{h \Delta \sigma_{\mathrm{sm}}}{2 \Delta \sigma_{\mathrm{sb}}}\right] \\ \sigma_{\mathrm{y}}-\frac{\Delta \sigma_{\mathrm{sm}}}{2}-\frac{\Delta \sigma_{\mathrm{sb}}}{h} y, & y \in\left[-\frac{h \Delta \sigma_{\mathrm{sm}}}{2 \Delta \sigma_{\mathrm{sb}}}, \frac{h}{2}\right]\end{cases}
$$

Case 2a corresponding the condition, $\Delta \sigma_{\mathrm{sm}}+\Delta \sigma_{\mathrm{sb}} \geq 2 \sigma_{\mathrm{y}}, \Delta \sigma_{\mathrm{sm}}-\Delta \sigma_{\mathrm{sb}} \leq 2 \sigma_{y}$ and $\Delta \sigma_{\mathrm{sm}} \geq \Delta \sigma_{\mathrm{sb}}$ :

$$
\sigma_{y}^{\prime}(y)=\left\{\begin{array}{cl}
\sigma_{\mathrm{y}}-\frac{\Delta \sigma_{\mathrm{sm}}}{2}-\frac{\Delta \sigma_{\mathrm{sb}}}{h} y, & y \in\left[-\frac{h}{2}, \frac{2 \sigma_{\mathrm{y}}-\Delta \sigma_{\mathrm{sm}}}{2 \Delta \sigma_{\mathrm{sb}}} h\right] \\
0 & y \in\left[\frac{2 \sigma_{\mathrm{y}}-\Delta \sigma_{\mathrm{sm}}}{2 \Delta \sigma_{\mathrm{sb}}} h, \frac{h}{2}\right]
\end{array}\right.
$$

Case $2 \mathbf{b}$ corresponding the condition $\Delta \sigma_{\mathrm{sm}}+\Delta \sigma_{\mathrm{sb}} \geq 2 \sigma_{\mathrm{y}}, \Delta \sigma_{\mathrm{sm}}-\Delta \sigma_{\mathrm{sb}} \leq 2 \sigma_{y}$ and $\Delta \sigma_{\mathrm{sm}} \leq \Delta \sigma_{\mathrm{sb}}$ :

$$
\sigma_{y}^{\prime}(y)=\left\{\begin{array}{cc}
\sigma_{\mathrm{y}}+\frac{\Delta \sigma_{\mathrm{sm}}}{2}+\frac{\Delta \sigma_{\mathrm{sb}}}{h} y, & y \in\left[-\frac{h}{2}, \frac{-\Delta \sigma_{\mathrm{sm}}}{2 \Delta \sigma_{\mathrm{sb}}} h\right] \\
\sigma_{\mathrm{y}}-\frac{\Delta \sigma_{\mathrm{sm}}}{2}-\frac{\Delta \sigma_{\mathrm{sb}}}{h} y, & y \in\left[\frac{-\Delta \sigma_{\mathrm{sm}}}{2 \Delta \sigma_{\mathrm{sb}}} h, \frac{2 \sigma_{\mathrm{y}}-\Delta \sigma_{\mathrm{sm}}}{2 \Delta \sigma_{\mathrm{sb}}} h\right] \\
0 & y \in\left[\frac{2 \sigma_{\mathrm{y}}-\Delta \sigma_{\mathrm{sm}}}{2 \Delta \sigma_{\mathrm{sb}}} h, \frac{h}{2}\right]
\end{array}\right.
$$

Case 3 corresponding the condition $\Delta \sigma_{\mathrm{sm}}+\Delta \sigma_{\mathrm{sb}} \geq 2 \sigma_{\mathrm{y}}, \Delta \sigma_{\mathrm{sb}}-\Delta \sigma_{\mathrm{sm}} \geq 2 \sigma_{y}$ and $\Delta \sigma_{\mathrm{sm}} \leq \Delta \sigma_{\mathrm{sb}}$ :

$$
\sigma_{y}^{\prime}(y)=\left\{\begin{array}{cc}
0 & y \in\left[-\frac{h}{2},-\frac{\Delta \sigma_{\mathrm{sm}}+2 \sigma_{\mathrm{y}}}{2 \Delta \sigma_{\mathrm{sb}}} h\right] \\
\sigma_{\mathrm{y}}+\frac{\Delta \sigma_{\mathrm{sm}}}{2}+\frac{\Delta \sigma_{\mathrm{sb}}}{h} y, & y \in\left[-\frac{\Delta \sigma_{\mathrm{sm}}+2 \sigma_{\mathrm{y}}}{2 \Delta \sigma_{\mathrm{sb}}} h,-\frac{\Delta \sigma_{\mathrm{sm}}}{2 \Delta \sigma_{\mathrm{sb}}} h\right] \\
\sigma_{\mathrm{y}}-\frac{\Delta \sigma_{\mathrm{sm}}}{2}-\frac{\Delta \sigma_{\mathrm{sb}}}{h} y, & y \in\left[\frac{-\Delta \sigma_{\mathrm{sm}}}{2 \Delta \sigma_{\mathrm{sb}}} h \frac{2 \sigma_{\mathrm{y}}-\Delta \sigma_{\mathrm{sm}}}{2 \Delta \sigma_{\mathrm{sb}}} h\right] \\
0 & y \in\left[\frac{2 \sigma_{\mathrm{y}}-\Delta \sigma_{\mathrm{sm}}}{2 \Delta \sigma_{\mathrm{sb}}} h, \frac{h}{2}\right]
\end{array}\right.
$$

Substituting the remaining yield stress $\sigma_{y}^{\prime}(y)$ for the five cases into the equilibrium equations Eqs. (5) and (6), then the mechanical membrane stress $\sigma_{\mathrm{pm}}$ and bending stress $\sigma_{\mathrm{pb}}$ are separately obtained as follows:

Case 1a , $\Delta \sigma_{\mathrm{sm}}+\Delta \sigma_{\mathrm{sb}} \leq 2 \sigma_{\mathrm{y}}$ and $\left|\Delta \sigma_{\mathrm{sm}}\right| \geq\left|\Delta \sigma_{\mathrm{sb}}\right|$ :

$$
\begin{aligned}
\sigma_{\mathrm{pm}} & =\frac{4 \Delta \sigma_{\mathrm{sb}} \bar{y}_{b}^{2}+\left(-8 \sigma_{\mathrm{y}}+4 \Delta \sigma_{\mathrm{sm}}\right) \bar{y}_{b}-\Delta \sigma_{\mathrm{sb}}}{4} \\
\sigma_{\mathrm{pb}} & =4 \Delta \sigma_{\mathrm{sb}} \bar{y}_{b}^{3}+\frac{3}{2} \sigma_{\mathrm{y}}-6 \sigma_{\mathrm{y}} \bar{y}_{b}^{2}-\frac{3}{4} \Delta \sigma_{\mathrm{sm}}+3 \Delta \sigma_{\mathrm{sm}} \bar{y}_{b}^{2}
\end{aligned}
$$


Case 1b, $\Delta \sigma_{\mathrm{sm}}+\Delta \sigma_{\mathrm{sb}} \leq 2 \sigma_{\mathrm{y}}$ and $\left|\Delta \sigma_{\mathrm{sm}}\right| \leq\left|\Delta \sigma_{\mathrm{sb}}\right|$ :

For: $\bar{y}_{b} \in\left[-\frac{\Delta \sigma_{\mathrm{sm}}}{2 \Delta \sigma_{\mathrm{sb}}}, \frac{1}{2}\right]$

$\sigma_{\mathrm{pm}}=\frac{4 \Delta \sigma_{\mathrm{sb}}^{2} \bar{y}_{b}^{2}+\left(4 \bar{y}_{b}-2\right) \Delta \sigma_{\mathrm{sm}} \Delta \sigma_{\mathrm{sb}}-8 \sigma_{\mathrm{y}} \bar{y}_{b} \Delta \sigma_{\mathrm{sb}}+\Delta \sigma_{\mathrm{sm}}^{2}}{4 \Delta \sigma_{\mathrm{sb}}}$

$\sigma_{\mathrm{pb}}=\frac{\left(16 \bar{y}_{b}^{3}-2\right) \Delta \sigma_{\mathrm{sb}}^{3}+\left(\left(-24 \sigma_{\mathrm{y}}+12 \Delta \sigma_{\mathrm{sm}}\right) \bar{y}_{b}^{2}+6 \sigma_{\mathrm{y}}\right) \Delta \sigma_{\mathrm{sb}}^{2}-\Delta \sigma_{\mathrm{sm}}^{3}}{4 \Delta \sigma_{\mathrm{sb}}^{2}}$

For: $\bar{y}_{b} \in\left[-\frac{1}{2},-\frac{\Delta \sigma_{\mathrm{sm}}}{2 \Delta \sigma_{\mathrm{sb}}}\right]$

$\sigma_{\mathrm{pm}}=\frac{-4 \Delta \sigma_{\mathrm{sb}}^{2} \bar{y}_{b}^{2}+\left(-4 \bar{y}_{b}-2\right) \Delta \sigma_{\mathrm{sm}} \Delta \sigma_{\mathrm{sb}}-8 \sigma_{\mathrm{y}} \bar{y}_{b} \Delta \sigma_{\mathrm{sb}}-\Delta \sigma_{\mathrm{sm}}^{2}}{4 \Delta \sigma_{\mathrm{sb}}}$

$\sigma_{\mathrm{pb}}=\frac{\left(-16 \bar{y}_{b}^{3}-2\right) \Delta \sigma_{\mathrm{sb}}^{3}+\left(\left(-24 \sigma_{\mathrm{y}}-12 \Delta \sigma_{\mathrm{sm}}\right) \bar{y}_{b}^{2}+6 \sigma_{\mathrm{y}}\right) \Delta \sigma_{\mathrm{sb}}^{2}+\Delta \sigma_{\mathrm{sm}}^{3}}{4 \Delta \sigma_{\mathrm{sb}}^{2}}$

Case 2a, $\Delta \sigma_{\mathrm{sm}}+\Delta \sigma_{\mathrm{sb}} \geq 2 \sigma_{\mathrm{y}}, \Delta \sigma_{\mathrm{sm}}-\Delta \sigma_{\mathrm{sb}} \leq 2 \sigma_{y}$ and $\Delta \sigma_{\mathrm{sm}} \geq \Delta \sigma_{\mathrm{sb}}$ :

$$
\begin{gathered}
\sigma_{\mathrm{pm}}=\frac{\Delta \sigma_{\mathrm{sb}}^{2}\left(8 \bar{y}_{b}^{2}-1\right)-16\left(\bar{y}_{b}+\frac{1}{4}\right)\left(\sigma_{\mathrm{y}}-\frac{\Delta \sigma_{\mathrm{sm}}}{2}\right) \Delta \sigma_{\mathrm{sb}}+4\left(\sigma_{\mathrm{y}}-\frac{\Delta \sigma_{\mathrm{sm}}}{2}\right)^{2}}{8 \Delta \sigma_{\mathrm{sb}}} \\
\sigma_{\mathrm{pb}}=\frac{\Delta \sigma_{\mathrm{sb}}^{3}\left(32 \bar{y}_{b}^{3}+2\right)-48\left(\bar{y}_{b}^{2}-\frac{1}{8}\right)\left(\sigma_{\mathrm{y}}-\frac{\Delta \sigma_{\mathrm{sm}}}{2}\right) \Delta \sigma_{\mathrm{sb}}^{2}+8\left(\sigma_{\mathrm{y}}-\frac{\Delta \sigma_{\mathrm{sm}}}{2}\right)^{3}}{8 \Delta \sigma_{\mathrm{sb}}^{2}}
\end{gathered}
$$

Case 2b, $\Delta \sigma_{\mathrm{sm}}+\Delta \sigma_{\mathrm{sb}} \geq 2 \sigma_{\mathrm{y}}, \Delta \sigma_{\mathrm{sm}}-\Delta \sigma_{\mathrm{sb}} \leq 2 \sigma_{y}$ and $\Delta \sigma_{\mathrm{sm}} \leq \Delta \sigma_{\mathrm{sb}}$ :

For: $\bar{y}_{b} \in\left[-\frac{\Delta \sigma_{\mathrm{sm}}}{2 \Delta \sigma_{\mathrm{sb}}}, \frac{2 \sigma_{\mathrm{y}}-\Delta \sigma_{\mathrm{sm}}}{2 \Delta \sigma_{\mathrm{sb}}}\right]$

$\sigma_{\mathrm{pm}}=\frac{1}{8 \Delta \sigma_{\mathrm{sb}}}\left[\begin{array}{l}\left(8 \bar{y}_{b}^{2}+1\right) \Delta \sigma_{\mathrm{sb}}^{2}+\left(\left(8 \bar{y}_{b}-2\right) \Delta \sigma_{\mathrm{sm}}+\left(-16 \bar{y}_{b}-4\right) \sigma_{\mathrm{y}}\right) \Delta \sigma_{\mathrm{sb}} \\ +4 \sigma_{\mathrm{y}}^{2}-4 \sigma_{\mathrm{y}} \Delta \sigma_{\mathrm{sm}}+3 \Delta \sigma_{\mathrm{sm}}^{2}\end{array}\right]$

$\sigma_{\mathrm{pm}}=\frac{1}{8 \Delta \sigma_{\mathrm{sb}}}\left[\begin{array}{l}\left(32 \bar{y}_{b}^{3}-2\right) \Delta \sigma_{\mathrm{sb}}^{3}+\left(24 \Delta \sigma_{\mathrm{sm}} \bar{y}_{b}^{2}-48 \sigma_{\mathrm{y}} \bar{y}_{b}^{2}+3 \Delta \sigma_{\mathrm{sm}}+6 \sigma_{\mathrm{y}}\right) \Delta \sigma_{\mathrm{sb}}^{2} \\ +8 \sigma_{\mathrm{y}}^{3}-12 \sigma_{\mathrm{y}}^{2} \Delta \sigma_{\mathrm{sm}}+6 \sigma_{\mathrm{y}} \Delta \sigma_{\mathrm{sm}}^{2}-3 \Delta \sigma_{\mathrm{sm}}^{3}\end{array}\right]$

For: $\bar{y}_{b} \in\left[-\frac{1}{2},-\frac{\Delta \sigma_{\mathrm{sm}}}{2 \Delta \sigma_{\mathrm{sb}}}\right]$

$\sigma_{\mathrm{pm}}=\frac{1}{8 \Delta \sigma_{\mathrm{sb}}}\left[\begin{array}{l}\left(-8 \bar{y}_{b}^{2}+1\right) \Delta \sigma_{\mathrm{sb}}^{2}-16\left(\bar{y}_{b}+\frac{1}{4}\right)\left(\sigma_{\mathrm{y}}+\frac{1}{2} \Delta \sigma_{\mathrm{sm}}\right) \Delta \sigma_{\mathrm{sb}} \\ +4 \sigma_{\mathrm{y}}^{2}-4 \sigma_{\mathrm{y}} \Delta \sigma_{\mathrm{sm}}-\Delta \sigma_{\mathrm{sm}}^{2}\end{array}\right]$ 


$$
\sigma_{\mathrm{pm}}=\frac{1}{8 \Delta \sigma_{\mathrm{sb}}}\left[\begin{array}{l}
\left(-32 \bar{y}_{b}^{3}-2\right) \Delta \sigma_{\mathrm{sb}}^{3}-48\left(\sigma_{\mathrm{y}}+\frac{1}{2} \Delta \sigma_{\mathrm{sm}}\right)\left(\bar{y}_{b}^{2}-\frac{1}{8}\right) \Delta \sigma_{\mathrm{sb}}^{2} \\
+8 \sigma_{\mathrm{y}}^{3}-12 \sigma_{\mathrm{y}}^{2} \Delta \sigma_{\mathrm{sm}}+6 \sigma_{\mathrm{y}} \Delta \sigma_{\mathrm{sm}}^{2}+\Delta \sigma_{\mathrm{sm}}^{3}
\end{array}\right]
$$

Case 3, $\Delta \sigma_{\mathrm{sm}}+\Delta \sigma_{\mathrm{sb}} \geq 2 \sigma_{\mathrm{y}}, \Delta \sigma_{\mathrm{sb}}-\Delta \sigma_{\mathrm{sm}} \geq 2 \sigma_{y}$ and $\Delta \sigma_{\mathrm{sm}} \leq \Delta \sigma_{\mathrm{sb}}$ :

$$
\begin{aligned}
& \text { For } \bar{y}_{b} \in\left[-\frac{\Delta \sigma_{\mathrm{sm}}}{2 \Delta \sigma_{\mathrm{sb}}}, \frac{2 \sigma_{\mathrm{y}}-\Delta \sigma_{\mathrm{sm}}}{2 \Delta \sigma_{\mathrm{sb}}}\right] \\
& \sigma_{\mathrm{pm}}=\frac{\left(2 \Delta \sigma_{\mathrm{sb}} \bar{y}_{b}+\Delta \sigma_{\mathrm{sm}}-4 \sigma_{\mathrm{y}}\right)\left(2 \Delta \sigma_{\mathrm{sb}} \bar{y}_{b}+\Delta \sigma_{\mathrm{sm}}\right)}{4 \Delta \sigma_{\mathrm{sb}}} \\
& \sigma_{\mathrm{pm}}=\frac{16 \Delta \sigma_{\mathrm{sb}}^{3} \bar{y}_{b}^{3}-24 \bar{y}_{b}^{2}\left(\sigma_{\mathrm{y}}-\frac{1}{2} \Delta \sigma_{\mathrm{sm}}\right) \Delta \sigma_{\mathrm{sb}}^{2}+8 \sigma_{\mathrm{y}}^{3}+6 \Delta \sigma_{\mathrm{sm}}^{2} \sigma_{\mathrm{y}}-\Delta \sigma_{\mathrm{sm}}^{3}}{4 \Delta \sigma_{\mathrm{sb}}^{2}} \\
& \text { For } \bar{y}_{b} \in\left[-\frac{2 \sigma_{\mathrm{y}}+\Delta \sigma_{\mathrm{sm}}}{2 \Delta \sigma_{\mathrm{sb}}},-\frac{\Delta \sigma_{\mathrm{sm}}}{2 \Delta \sigma_{\mathrm{sb}}}\right] \\
& \sigma_{\mathrm{pm}}=-\frac{\left(2 \Delta \sigma_{\mathrm{sb}} \bar{y}_{b}+\Delta \sigma_{\mathrm{sm}}+4 \sigma_{\mathrm{y}}\right)\left(2 \Delta \sigma_{\mathrm{sb}} \bar{y}_{b}+\Delta \sigma_{\mathrm{sm}}\right)}{4 \Delta \sigma_{\mathrm{sb}}} \\
& -16 \Delta \sigma_{\mathrm{sb}}^{3} \bar{y}_{b}^{3}-24 \bar{y}_{b}^{2}\left(\sigma_{\mathrm{y}}+\frac{1}{2} \Delta \sigma_{\mathrm{sm}}\right) \Delta \sigma_{\mathrm{sb}}^{2} \\
& \sigma_{\mathrm{pm}}=\frac{+8 \sigma_{\mathrm{y}}^{3}+6 \Delta \sigma_{\mathrm{sm}}^{2} \sigma_{\mathrm{y}}+\Delta \sigma_{\mathrm{sm}}^{3}}{4 \Delta \sigma_{\mathrm{sb}}^{2}}
\end{aligned}
$$

where the dimensionless parameter $\bar{y}_{b}=y_{b} / h$.

Through eliminating $y_{b}$ in Eqs. (12) (27), respectively, the mechanical bending stress $\sigma_{\mathrm{pb}}$ corresponding to the limit state can be expressed in terms of the mechanical membrane stress $\sigma_{\mathrm{pm}}$ as follows:

Case 1a , $\Delta \sigma_{\mathrm{sm}}+\Delta \sigma_{\mathrm{sb}} \leq 2 \sigma_{\mathrm{y}}$ and $\left|\Delta \sigma_{\mathrm{sm}}\right| \geq\left|\Delta \sigma_{\mathrm{sb}}\right|$ :

$$
\sigma_{\mathrm{pb}}=\frac{3 b^{2} \sigma_{\mathrm{pm}}-3 a^{2} \sigma_{\mathrm{pm}}-\sqrt{2}\left(a^{2}+b^{2}-2 a \sigma_{\mathrm{pm}}+2 b \sigma_{\mathrm{pm}}\right)^{\frac{3}{2}}+2\left(a^{3}+b^{3}\right)}{(a-b)^{2}}
$$

where:

$$
\begin{aligned}
& a=\sigma_{\mathrm{y}}-\frac{\Delta \sigma_{\mathrm{sm}}+\Delta \sigma_{\mathrm{sb}}}{2} \\
& \mathrm{~b}=\sigma_{\mathrm{y}}-\frac{\Delta \sigma_{\mathrm{sm}}-\Delta \sigma_{\mathrm{sb}}}{2}
\end{aligned}
$$


Case 1b, $\Delta \sigma_{\mathrm{sm}}+\Delta \sigma_{\mathrm{sb}} \leq 2 \sigma_{\mathrm{y}}$ and $\left|\Delta \sigma_{\mathrm{sm}}\right| \leq\left|\Delta \sigma_{\mathrm{sb}}\right|$ :

$$
\begin{aligned}
& \text { For } \sigma_{\mathrm{pm}} \leq \frac{\left(2 \sigma_{y}-\Delta \sigma_{\mathrm{sb}}\right) \Delta \sigma_{\mathrm{sm}}}{2 \Delta \sigma_{\mathrm{sb}}}: \\
& \sigma_{\mathrm{pb}}=-\frac{1}{2 \Delta \sigma_{\mathrm{sb}}^{2}}\left[\begin{array}{l}
2 \sqrt{2}\left(\begin{array}{l}
\left.\left(\Delta \sigma_{\mathrm{sm}}+2 \sigma_{\mathrm{pm}}\right) \Delta \sigma_{\mathrm{sb}}\right)^{\frac{3}{2}} \\
+2 \sigma_{\mathrm{y}}\left(\sigma_{\mathrm{y}}-\Delta \sigma_{\mathrm{sm}}\right)
\end{array}\right)+\Delta \sigma_{\mathrm{sb}}^{3}-3 \Delta \sigma_{\mathrm{sb}}^{2} \sigma_{\mathrm{y}} \\
-6\left(\Delta \sigma_{\mathrm{sm}}+2 \sigma_{\mathrm{pm}}\right)\left(\sigma_{\mathrm{y}}-\frac{1}{2} \Delta \sigma_{\mathrm{sm}}\right) \Delta \sigma_{\mathrm{sb}}-8 \sigma_{\mathrm{y}}^{3}+12 \Delta \sigma_{\mathrm{sm}} \sigma_{\mathrm{y}}^{2}-3 \Delta \sigma_{\mathrm{sm}}^{2} \sigma_{\mathrm{y}}
\end{array}\right] \\
& \left.\begin{array}{c}
\text { For } \sigma_{\mathrm{pm}}>\frac{\left(2 \sigma_{y}-\Delta \sigma_{\mathrm{sb}}\right) \Delta \sigma_{\mathrm{sm}}}{2 \Delta \sigma_{\mathrm{sb}}}: \\
\sigma_{\mathrm{pb}}=-\frac{1}{2 \Delta \sigma_{\mathrm{sb}}^{2}}\left[\begin{array}{l}
\left(-\Delta \sigma_{\mathrm{sm}}-2 \sigma_{\mathrm{pm}}\right) \Delta \sigma_{\mathrm{sb}} \\
2 \sqrt{2} \\
+2 \sigma_{\mathrm{y}}\left(\sigma_{\mathrm{y}}+\Delta \sigma_{\mathrm{sm}}\right)
\end{array}\right)^{\frac{3}{2}}+\Delta \sigma_{\mathrm{sb}}^{3}-3 \Delta \sigma_{\mathrm{sb}}^{2} \sigma_{\mathrm{y}} \\
+6\left(\Delta \sigma_{\mathrm{sm}}+2 \sigma_{\mathrm{pm}}\right)\left(\sigma_{\mathrm{y}}+\frac{1}{2} \Delta \sigma_{\mathrm{sm}}\right) \Delta \sigma_{\mathrm{sb}}-8 \sigma_{\mathrm{y}}^{3}-12 \Delta \sigma_{\mathrm{sm}} \sigma_{\mathrm{y}}^{2}-3 \Delta \sigma_{\mathrm{sm}}^{2} \sigma_{\mathrm{y}}
\end{array}\right]
\end{aligned}
$$

Case $2 \mathrm{a}, \Delta \sigma_{\mathrm{sm}}+\Delta \sigma_{\mathrm{sb}} \geq 2 \sigma_{\mathrm{y}}, \Delta \sigma_{\mathrm{sm}}-\Delta \sigma_{\mathrm{sb}} \leq 2 \sigma_{y}$ and $\Delta \sigma_{\mathrm{sm}} \geq \Delta \sigma_{\mathrm{sb}}$ :

$$
\sigma_{\mathrm{pb}}=-\frac{1}{8 \Delta \sigma_{\mathrm{sb}}^{2}}\left[\begin{array}{l}
\sqrt{2}\left(\Delta \sigma_{\mathrm{sb}}^{2}+\left(4 \sigma_{\mathrm{y}}-2 \Delta \sigma_{\mathrm{sm}}+8 \sigma_{\mathrm{pm}}\right) \Delta \sigma_{\mathrm{sb}}+4\left(\sigma_{\mathrm{y}}-\frac{1}{2} \Delta \sigma_{\mathrm{sm}}\right)^{2}\right)^{\frac{3}{2}}+2 \Delta \sigma_{\mathrm{sb}}^{3} \\
+\left(12 \sigma_{\mathrm{y}}-6 \Delta \sigma_{\mathrm{sm}}\right) \Delta \sigma_{\mathrm{sb}}^{2}+24\left(\sigma_{\mathrm{y}}-\frac{1}{2} \Delta \sigma_{\mathrm{sm}}\right)\left(\sigma_{\mathrm{y}}-\frac{1}{2} \Delta \sigma_{\mathrm{sm}}+2 \sigma_{\mathrm{pm}}\right) \Delta \sigma_{\mathrm{sb}} \\
+16\left(\sigma_{\mathrm{y}}-\frac{1}{2} \Delta \sigma_{\mathrm{sm}}\right)^{3}
\end{array}\right]
$$

Case 2b, $\Delta \sigma_{\mathrm{sm}}+\Delta \sigma_{\mathrm{sb}} \geq 2 \sigma_{\mathrm{y}}, \Delta \sigma_{\mathrm{sb}}-\Delta \sigma_{\mathrm{sm}} \leq 2 \sigma_{y}$ and $\Delta \sigma_{\mathrm{sm}} \leq \Delta \sigma_{\mathrm{sb}}$ :

$$
\begin{aligned}
& \text { For } \sigma_{\mathrm{pm}} \leq \frac{\left(\Delta \sigma_{\mathrm{sb}}-\Delta \sigma_{\mathrm{sm}}-2 \sigma_{y}\right)^{2}}{8 \Delta \sigma_{\mathrm{sb}}}: \\
& \sigma_{\mathrm{pb}}=-\frac{1}{8 \Delta \sigma_{\mathrm{sb}}^{2}}\left[\begin{array}{l}
\sqrt{2}\left(-\Delta \sigma_{\mathrm{sb}}^{2}+\left(4 \sigma_{\mathrm{y}}+2 \Delta \sigma_{\mathrm{sm}}+8 \sigma_{\mathrm{pm}}\right) \Delta \sigma_{\mathrm{sb}}+4 \sigma_{\mathrm{y}}^{2}-4 \sigma_{\mathrm{y}} \Delta \sigma_{\mathrm{sm}}-\Delta \sigma_{\mathrm{sm}}^{2}\right)^{\frac{3}{2}} \\
+2 \Delta \sigma_{\mathrm{sb}}^{3}-6 \Delta \sigma_{\mathrm{sb}}^{2} \Delta \sigma_{\mathrm{sm}}-24\left(\sigma_{\mathrm{y}}-\frac{1}{2} \Delta \sigma_{\mathrm{sm}}\right)\left(\sigma_{\mathrm{y}}+\frac{1}{2} \Delta \sigma_{\mathrm{sm}}+2 \sigma_{\mathrm{pm}}\right) \Delta \sigma_{\mathrm{sb}} \\
-16 \sigma_{\mathrm{y}}^{3}+24 \Delta \sigma_{\mathrm{sm}} \sigma_{\mathrm{y}}^{2}-2 \Delta \sigma_{\mathrm{sm}}^{3}
\end{array}\right] \\
& \text { For } \sigma_{\mathrm{pm}} \geq \frac{\left(\Delta \sigma_{\mathrm{sb}}-\Delta \sigma_{\mathrm{sm}}-2 \sigma_{y}\right)^{2}}{8 \Delta \sigma_{\mathrm{sb}}}:
\end{aligned}
$$




$$
\sigma_{\mathrm{pb}}=\frac{1}{8 \Delta \sigma_{\mathrm{sb}}^{2}}\left[\begin{array}{l}
-\sqrt{2}\left(\begin{array}{l}
\left.\Delta \sigma_{\mathrm{sb}}^{2}+\left(-4 \sigma_{\mathrm{y}}-2 \Delta \sigma_{\mathrm{sm}}-8 \sigma_{\mathrm{pm}}\right) \Delta \sigma_{\mathrm{sb}}\right)^{\frac{3}{2}} \\
+12 \sigma_{\mathrm{y}}^{2}+4 \sigma_{\mathrm{y}} \Delta \sigma_{\mathrm{sm}}+\Delta \sigma_{\mathrm{sm}}^{2}
\end{array}\right. \\
-2 \Delta \sigma_{\mathrm{sb}}^{3}+\left(12 \sigma_{\mathrm{y}}+6 \Delta \sigma_{\mathrm{sm}}\right) \Delta \sigma_{\mathrm{sb}}^{2} \\
-24\left(\sigma_{\mathrm{y}}+\frac{1}{2} \Delta \sigma_{\mathrm{sm}}\right)\left(\sigma_{\mathrm{y}}+2 \sigma_{\mathrm{pm}}+\frac{1}{2} \Delta \sigma_{\mathrm{sm}}\right) \Delta \sigma_{\mathrm{sb}} \\
+64 \sigma_{\mathrm{y}}^{3}+24 \Delta \sigma_{\mathrm{sm}} \sigma_{\mathrm{y}}^{2}+12 \Delta \sigma_{\mathrm{sm}}^{2} \sigma_{\mathrm{y}}+2 \Delta \sigma_{\mathrm{sm}}^{3}
\end{array}\right]
$$

Case 3, $\Delta \sigma_{\mathrm{sm}}+\Delta \sigma_{\mathrm{sb}} \geq 2 \sigma_{\mathrm{y}}, \Delta \sigma_{\mathrm{sb}}-\Delta \sigma_{\mathrm{sm}} \geq 2 \sigma_{y}$ and $\Delta \sigma_{\mathrm{sm}} \leq \Delta \sigma_{\mathrm{sb}}$ :

$$
\sigma_{\mathrm{pb}}=-\frac{4\left(\sigma_{\mathrm{y}}^{2}-\Delta \sigma_{\mathrm{sb}} \sigma_{\mathrm{pm}}\right)^{\frac{3}{2}}+\left(6 \sigma_{\mathrm{y}}+3 \Delta \sigma_{\mathrm{sm}}\right) \sigma_{\mathrm{pm}} \Delta \sigma_{\mathrm{sb}}-6 \sigma_{\mathrm{y}}^{3}}{\Delta \sigma_{\mathrm{sb}}^{2}}
$$

\subsection{Reduction to special cases}

For the special case when the beam is subjected to the cyclic thermal membrane stress $\sigma_{\text {sm }}$, and to a combination of the steady mechanical bending stress $\sigma_{\mathrm{pb}}$ and mechanical membrane stress $\sigma_{\mathrm{pm}}$. It can be found from Figure 3 that only the case (1a) can be degenerated into this situation $\Delta \sigma_{\mathrm{sb}}=0$. Then the expression of the ratchet boundary is reduced from Eq.(28) as the following :

$$
\sigma_{\mathrm{pb}}=\frac{3}{4}\left(2 \sigma_{y}-\Delta \sigma_{\mathrm{sm}}\right)+\frac{3 \sigma_{\mathrm{pm}}^{2}}{\Delta \sigma_{\mathrm{sm}}-2 \sigma_{y}}
$$

For another special case where the cyclic thermal bending stress $\sigma_{\mathrm{sb}}$, the steady mechanical bending stress $\sigma_{\mathrm{pb}}$ and membrane stress $\sigma_{\mathrm{pm}}$ act simultaneously, then the case (1b) and case (3) in Figure 3 can be degenerated into this situation $\Delta \sigma_{\mathrm{sm}}=0$. And the expressions of the ratchet boundary are reduced from Eqs. (32) and (36) as the following:

For $\Delta \sigma_{\mathrm{sb}} \leq 2 \sigma_{\mathrm{y}}$ :

$$
\sigma_{\mathrm{pb}}=\frac{1}{2 \Delta \sigma_{\mathrm{sb}}^{2}}\left[-8\left(-\sigma_{\mathrm{pm}} \Delta \sigma_{\mathrm{sb}}+\sigma_{y}^{2}\right)^{\frac{3}{2}}-12 \sigma_{\mathrm{y}} \Delta \sigma_{\mathrm{sb}} \sigma_{\mathrm{pm}}+3 \Delta \sigma_{\mathrm{sb}}^{2} \sigma_{\mathrm{y}}+8 \sigma_{\mathrm{y}}^{3}-\Delta \sigma_{\mathrm{sb}}^{3}\right]
$$

And for $\Delta \sigma_{\mathrm{sb}} \geq 2 \sigma_{\mathrm{y}}$ :

$$
\sigma_{\mathrm{pb}}=-\frac{4\left(\sigma_{\mathrm{y}}^{2}-\Delta \sigma_{\mathrm{sb}} \sigma_{\mathrm{pm}}\right)^{\frac{3}{2}}+6 \sigma_{\mathrm{y}} \sigma_{\mathrm{pm}} \Delta \sigma_{\mathrm{sb}}-6 \sigma_{\mathrm{y}}^{3}}{\Delta \sigma_{\mathrm{sb}}^{2}}
$$

\section{Discussion of Analytical Results}

\subsection{Thermal stresses including the thermal bending and membrane stress}


As it can be seen from Figure 3, it is clear that the allowable mechanical membrane stresses at the ratchet boundary will reach the maximum when the condition is satisfied as: $\sigma_{\mathrm{pm}}=\frac{1}{h} \int_{-\frac{h}{2}}^{\frac{h}{2}} \sigma_{y}^{\prime}(y) d y$

Thus, the detail expressions of Eq.(40) for the five cases in Figure 3 can be derivate as follows[8]:

Case 1a , $\Delta \sigma_{\mathrm{sm}}+\Delta \sigma_{\mathrm{sb}} \leq 2 \sigma_{\mathrm{y}}$ and $\left|\Delta \sigma_{\mathrm{sm}}\right| \geq\left|\Delta \sigma_{\mathrm{sb}}\right|$ :

$$
\sigma_{\mathrm{pm}}=\sigma_{\mathrm{y}}-\frac{\Delta \sigma_{\mathrm{sm}}}{2}
$$

Case 1b, $\Delta \sigma_{\mathrm{sm}}+\Delta \sigma_{\mathrm{sb}} \leq 2 \sigma_{\mathrm{y}}$ and $\left|\Delta \sigma_{\mathrm{sm}}\right| \leq\left|\Delta \sigma_{\mathrm{sb}}\right|$ :

$$
\sigma_{\mathrm{pm}}=\sigma_{\mathrm{y}}-\frac{\Delta \sigma_{\mathrm{sb}}}{4}-\frac{\Delta \sigma_{\mathrm{sm}}^{2}}{4 \Delta \sigma_{\mathrm{sb}}}
$$

Case 2a, $\Delta \sigma_{\mathrm{sm}}+\Delta \sigma_{\mathrm{sb}} \geq 2 \sigma_{\mathrm{y}}, \Delta \sigma_{\mathrm{sm}}-\Delta \sigma_{\mathrm{sb}} \leq 2 \sigma_{y}$ and $\Delta \sigma_{\mathrm{sm}} \geq \Delta \sigma_{\mathrm{sb}}$ :

$$
\sigma_{\mathrm{pm}}=\frac{\left[\sigma_{\mathrm{y}}-\frac{1}{2}\left(\Delta \sigma_{\mathrm{sm}}-\Delta \sigma_{\mathrm{sb}}\right)\right]^{2}}{2 \Delta \sigma_{\mathrm{sb}}}
$$

Case $2 \mathrm{~b}, \Delta \sigma_{\mathrm{sm}}+\Delta \sigma_{\mathrm{sb}} \geq 2 \sigma_{\mathrm{y}}, \Delta \sigma_{\mathrm{sb}}-\Delta \sigma_{\mathrm{sm}} \leq 2 \sigma_{y}$ and $\Delta \sigma_{\mathrm{sm}} \leq \Delta \sigma_{\mathrm{sb}}$ :

$$
\sigma_{\mathrm{pm}}=\frac{\left[\sigma_{\mathrm{y}}-\frac{1}{2}\left(\Delta \sigma_{\mathrm{sm}}-\Delta \sigma_{\mathrm{sb}}\right)\right]^{2}}{2 \Delta \sigma_{\mathrm{sb}}}-\frac{\left(\Delta \sigma_{\mathrm{sm}}-\Delta \sigma_{\mathrm{sb}}\right)^{2}}{4 \Delta \sigma_{\mathrm{sb}}}
$$

Case 3, $\Delta \sigma_{\mathrm{sm}}+\Delta \sigma_{\mathrm{sb}} \geq 2 \sigma_{\mathrm{y}}, \Delta \sigma_{\mathrm{sb}}-\Delta \sigma_{\mathrm{sm}} \geq 2 \sigma_{y}$ and $\Delta \sigma_{\mathrm{sm}} \leq \Delta \sigma_{\mathrm{sb}}$ :

$$
\sigma_{\mathrm{pm}}=\frac{\sigma_{\mathrm{y}}^{2}}{\Delta \sigma_{\mathrm{sb}}}
$$

According to Eqs. (41) (45), the ratchet boundary for the maximum allowable mechanical membrane stress $\sigma_{\mathrm{pm}}$ with the thermal membrane stress $\sigma_{\mathrm{sm}}$ and bending stress $\sigma_{\mathrm{sb}}$ can be depicted as three-dimensional shaded surface in Figure 4. 


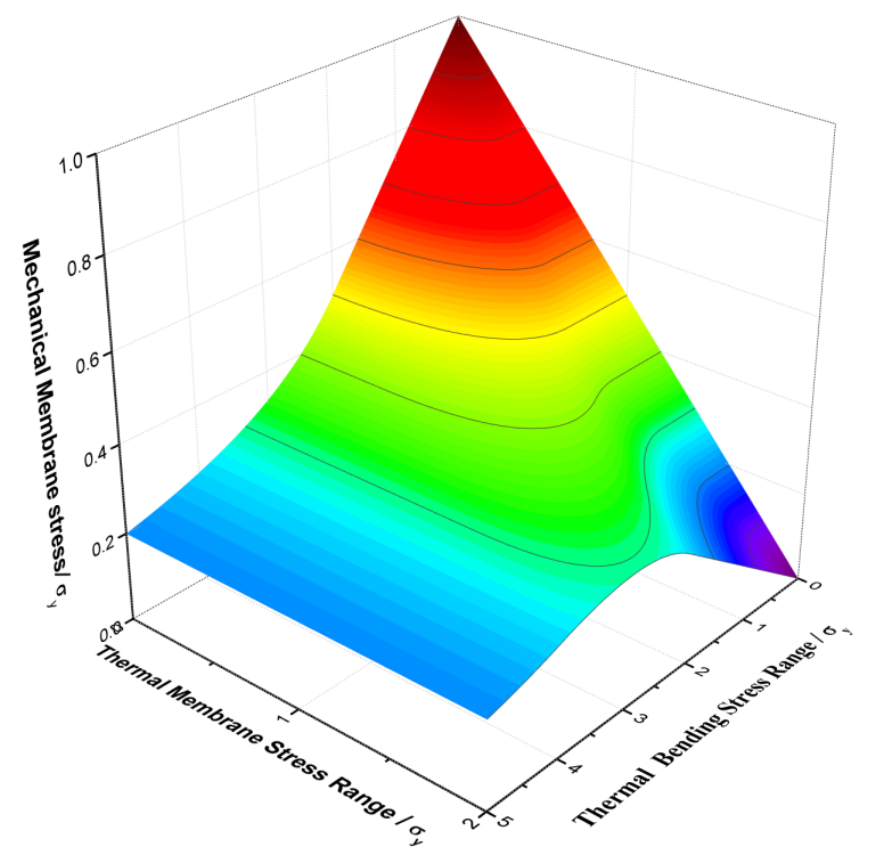

Figure 4. Ratchet boundary for the interaction of a mechanical membrane stress with cyclic thermal membrane and cyclic thermal bending stress

The results for the maximum allowable mechanical membrane stresses are consistent with Wolf Reinhardt's work [8], which however ignored the influence of the mechanical bending stress $\sigma_{\mathrm{pb}}$ and the Eqs.(41) (45) of which were not established on the equilibrium equation.

In current paper, based on the equilibrium equations (5) and (6), when the allowable mechanical membrane stresses reach the maximum, it is evident that the allowable mechanical bending stress in this case will not be reduced to zero unless the distribution of the remaining yield stress $\sigma_{y}^{\prime}(y)$ is symmetric about the neutral axial. Therefore, it may be not reasonable in Wolf Reinhardt's work [8] to ignore the effect of the mechanical bending stress when the maximum mechanical membrane stress is reached.

Corresponding to the maximum mechanical membrane stresses, the allowable mechanical bending stress is obtained by the equilibrium equation for mechanical bending stress in Eq.(6). Then the relationship of the allowable mechanical bending stress $\sigma_{\mathrm{pb}}$ with the thermal membrane stress $\sigma_{\mathrm{sm}}$ and bending stress $\sigma_{\mathrm{sb}}$ is presented in Figure 5. It can be found from Figure 5 that the allowable mechanical bending stress corresponding to the maximum mechanical membrane stresses is reduced to zero when the thermal stress only includes the thermal bending stress or thermal membrane stress. However, for the combination of the thermal bending stress and thermal membrane stress, the allowable mechanical bending stress is negative, which means the direction of the mechanical bending moment is changed. Besides, it further indicates that the mechanical bending stress can promote the load carrying capacity of the structure for the mechanical membrane stress sometimes. 


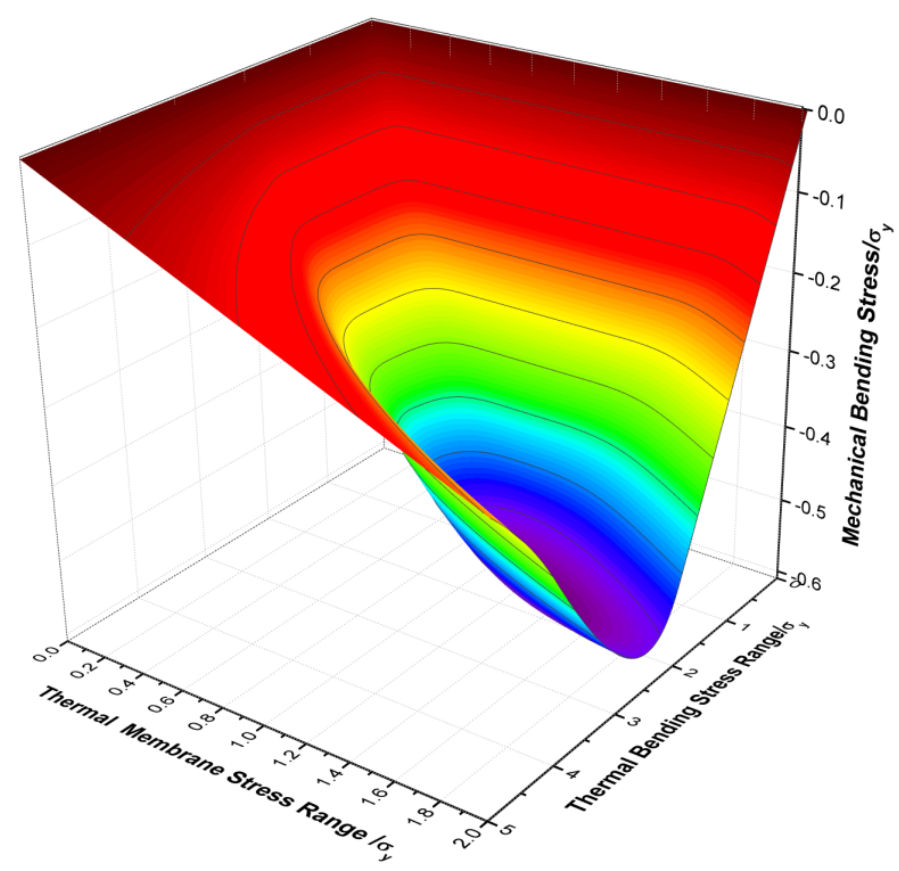

Figure 5. Ratchet boundary for the interaction of a mechanical bending stress with cyclic thermal membrane and cyclic thermal bending stress when the mechanical membrane stresses reach maximum

As can be seen from the above, when the mechanical stress includes both membrane and bending stress simultaneously, the mechanical bending stress shown in Figure 5 for the ratchet boundary leads to the maximum allowable mechanical membrane stress depicted in Figure 4.

In order to further compare the difference of the mechanical membrane stress $\sigma_{\mathrm{pm}}$ at ratchet boundary between the results in Wolf Reinhardt's work [8] and the results in current paper when the mechanical bending stress $\sigma_{\mathrm{pb}}$ reduces to zero, the mechanical membrane stress $\sigma_{\mathrm{pm}}$ at ratchet boundary for $\sigma_{\mathrm{pb}}=0$ is determined from equilibrium equations (5) and (6), which is shown in Figure 6. Taking comparison between Figure 6 and Wolf Reinhardt's result in Figure 4, the distinct difference between both figures can be found that for the large thermal bending stress (e.g. $\Delta \sigma_{\mathrm{sb}} / \sigma_{y}=5$,), the mechanical membrane stress decreases with the increment of the thermal membrane stress in Figure 6 while the mechanical membrane stress is independent of the thermal membrane stress in Figure 4 which corresponds to the case 3 in Figure 3 and equation (45). Furthermore, the difference between Figure 4 and Figure 6 is vividly depicted in Figure 7 by subtracting Figure 4 from Figure 6. As shown in Figure 7, the solution in Figure 6 is more conservative to the results in Figure 4, which means the derived analytical expressions are more conservative to the results in Ref [8] due to the equilibrium equation considered in current paper. Meanwhile, it can be found further that the maximum distinction corresponds to the case $2 \mathrm{a}$ and $2 \mathrm{~b}$ in Figure 3. Nevertheless, when the thermal membrane stress $\sigma_{\mathrm{sm}}$ or thermal bending stress $\sigma_{\mathrm{sb}}$ are reduced to zero, the difference disappears and the ratchet boundary derived in current paper is consistent with the results in Wolf Reinhardt's work [8], which attributes to the symmetric distribution of the new yield stress $\sigma_{y}^{\prime}(y)$ about 
the neutral axis at this time. Herein, for the case when the thermal membrane stress $\sigma_{\mathrm{sm}}$ is reduced to zero, the relationship between the mechanical membrane stress and the thermal bending stress in Figure 6 becomes into the classical Bree problem as expressed in [13]:

$$
\begin{gathered}
\sigma_{\mathrm{pm}}=\sigma_{y}-\frac{1}{4} \Delta \sigma_{\mathrm{sb}}, \quad \Delta \sigma_{\mathrm{sb}} \leq 2 \sigma_{y} \\
\sigma_{\mathrm{pm}}=\frac{\sigma_{y}^{2}}{\Delta \sigma_{\mathrm{sb}}}, \quad \Delta \sigma_{\mathrm{sb}}>2 \sigma_{y}
\end{gathered}
$$

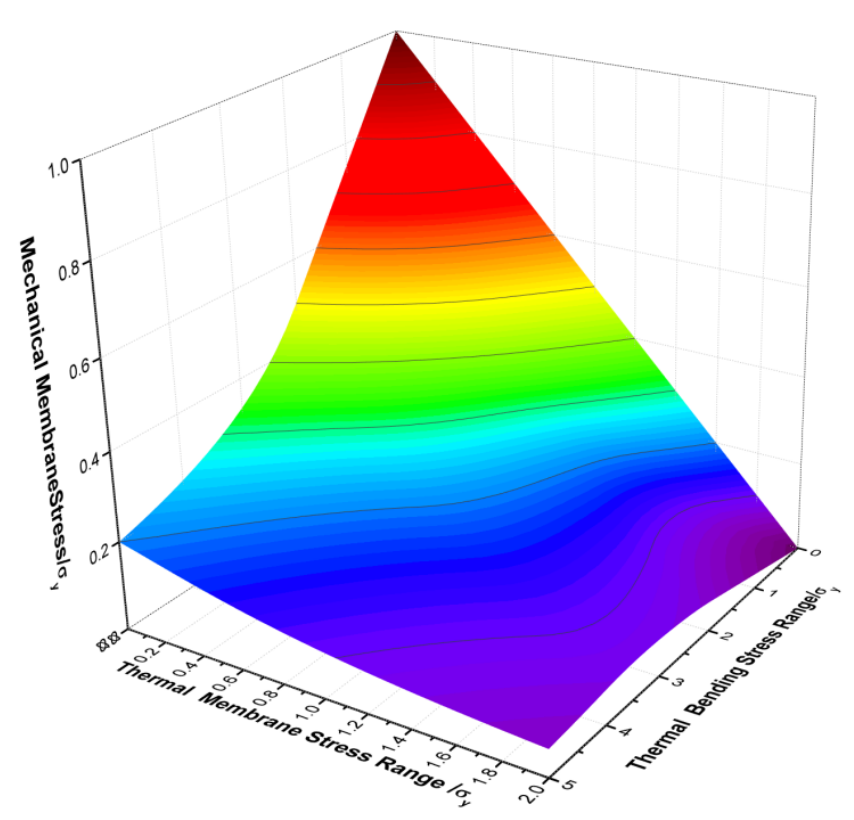

Figure 6. Ratchet boundary for the interaction of a mechanical membrane stress with cyclic thermal membrane and cyclic thermal bending stress when the mechanical bending stresses are reduced to zero 


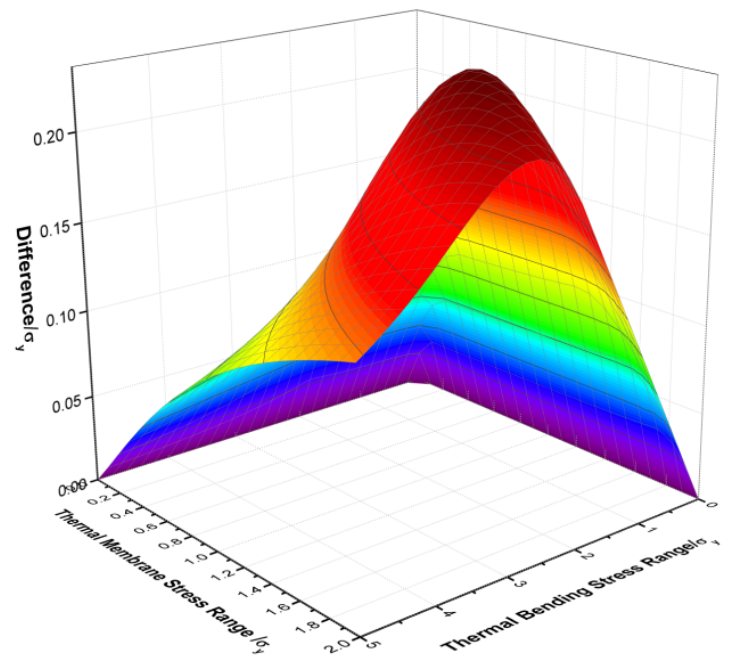

Figure 7. Difference between Figure 4 and Figure 6 by subtracting Figure 6 from Figure 4

On the other hand, when the mechanical membrane stress $\sigma_{\mathrm{pm}}$ is reduced to zero, the mechanical bending stress $\sigma_{\mathrm{pb}}$ in this case can be also obtained from equilibrium equations (5) and (6), as shown in Figure 8. Taking comparison between Figure 5 and Figure 8, it can be found that for some certain combination of thermal bending $\sigma_{\mathrm{sb}}$ and membrane stresses $\sigma_{\mathrm{sm}}$, the absolute value of mechanical bending stress corresponding to $\sigma_{\mathrm{pm}}=0$ in Figure 8 is lower than the absolute value of mechanical bending stress in Figure 5 which corresponds to the maximum mechanical membrane stress shown in Figure 4. Thus, it is indicated that for some certain combination of thermal bending $\sigma_{\mathrm{sb}}$ and membrane stresses $\sigma_{\mathrm{sm}}$, the mechanical membrane stress can also promote the load carrying capacity of the structure for the mechanical bending stress.

Furthermore, as the thermal bending stress $\sigma_{\mathrm{sb}}$ is reduced to zero, the ratchet boundary in Figure 8 for the interaction of the mechanical bending stress with the cyclic thermal membrane stress is degenerated into the inverse Bree problem ${ }^{8}$, where the mechanical bending stress is expressed as:

$$
\sigma_{\mathrm{pb}}=\frac{3}{2} \sigma_{y}-\frac{3}{4} \Delta \sigma_{\mathrm{sm}}
$$

And when the thermal membrane stress $\sigma_{\mathrm{sm}}$ is reduced to zero, the ratchet boundary in Figure 8 for the interaction of the mechanical bending stress with the cyclic thermal bending stress is degenerated as the expressions form ${ }^{3}$ :

$$
\begin{gathered}
\sigma_{\mathrm{pb}}=\frac{3}{2} \sigma_{y}-\frac{\Delta \sigma_{\mathrm{sb}}}{2}, \quad \Delta \sigma_{\mathrm{sb}} \leq 2 \sigma_{y} \\
\sigma_{\mathrm{pb}}=\frac{2 \sigma_{y}^{3}}{\Delta \sigma_{\mathrm{sb}}^{2}}, \quad \Delta \sigma_{\mathrm{sb}}>2 \sigma_{y}
\end{gathered}
$$




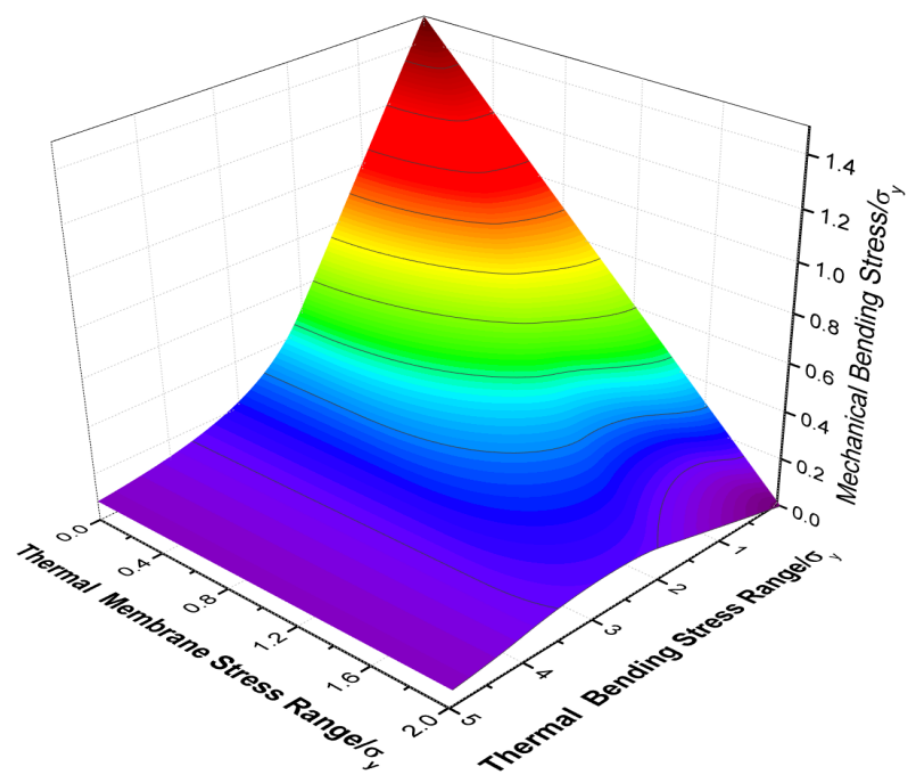

Figure 8. Ratchet boundary for the interaction of a mechanical bending stress with cyclic thermal membrane and cyclic thermal bending stress when the mechanical membrane stresses are reduced to zero

\subsection{Thermal stresses only including the thermal membrane stress}

For the special case where the cyclic thermal membrane stress $\sigma_{\mathrm{sm}}$, the steady mechanical bending $\sigma_{\mathrm{pb}}$ and membrane stress $\sigma_{\mathrm{pm}}$ act simultaneously, the relationships among the three kinds of stresses are depicted in Figure 9 from the Eq.(37). As it can be seen from the Figure 9, when the thermal stress only includes the thermal membrane stress, the increment of the mechanical membrane stress causes the load carrying capacity of the structure for the mechanical bending stress decreasing and vice versa. 


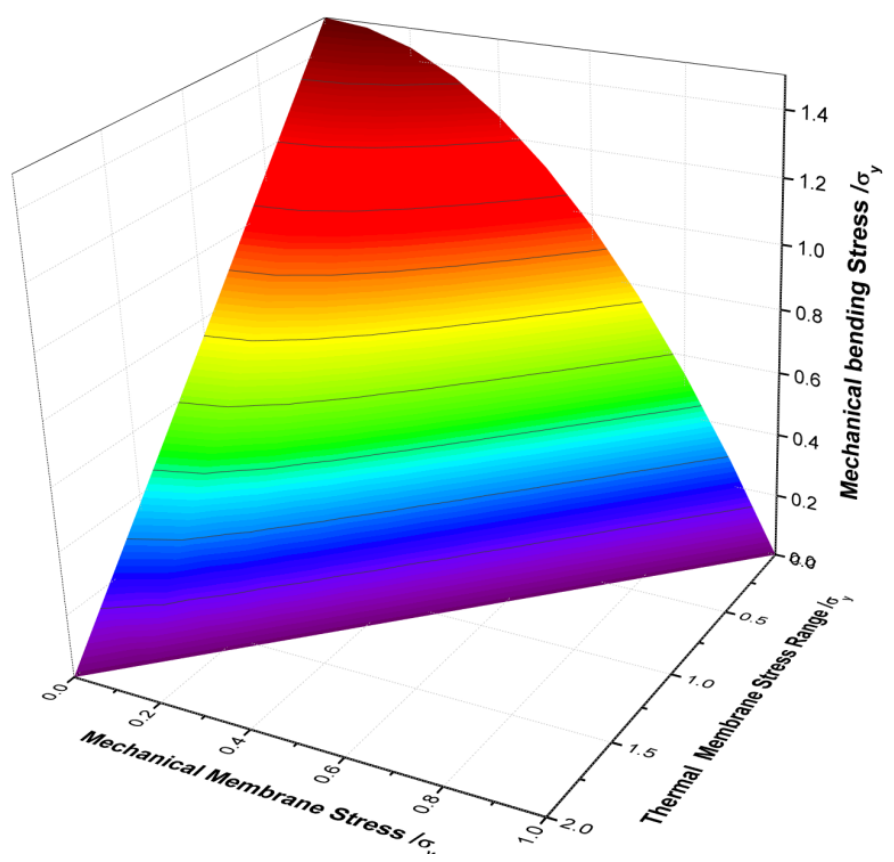

Figure 9. Ratchet boundary for the interaction of a mechanical bending stress with mechanical membrane stress and cyclic thermal membrane stress when the cyclic thermal bending stress is omitted

\subsection{Thermal stresses only including the thermal bending stress}

Similarly, when the thermal stress only includes the thermal bending stress $\sigma_{\mathrm{sb}}$, the figurative expression for the ratchet boundary of mechanical bending stress $\sigma_{\mathrm{pb}}$ with the mechanical membrane stress $\sigma_{\mathrm{pm}}$ and the thermal bending stress $\sigma_{\mathrm{sb}}$ can be presented in Figure 10 based on the equations (38) and (39). It can be found from Figure 10 that when the thermal stresses only includes the thermal bending stress, the load capacity for the mechanical bending stress decreases by increasing the mechanical membrane stress and vice versa. 


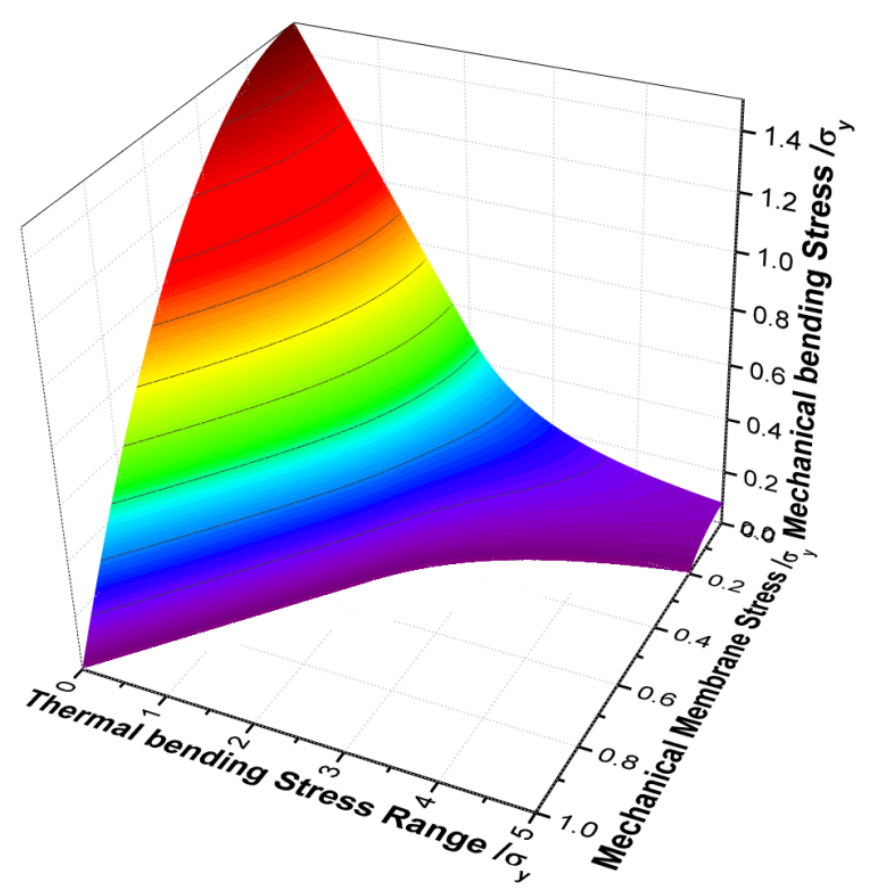

Figure 10. Ratchet boundary for the interaction of a mechanical bending stress with mechanical membrane stress and cyclic thermal bending stress when the cyclic thermal membrane stress is omitted

\section{Verification by finite element results}

\subsection{Two-plane model}

Several kinds of mechanical models were proposed to verify the correctness of Bree problem or similar problems. The two-bar model was used by Kalnins[14], Yukinori et al.[15]. These models have a common feature that rigid boundary subjected to mechanical load is set parallel to the fixed boundary. That means the rigid boundary cannot rotate. And because of this, mechanical moment cannot be imposed and the effect of mechanical bending stress on the ratcheting cannot be considered in these kinds of models.

In order to validate the correctness of the four-dimensional ratcheting boundary, a novel two-plane model was built as shown in Fig 11. It is a plane stress model consisted in two planes posed face to face. Displacement-freedom in Y-direction of two outside boundary lines was restrained. Two inside boundary lines were kept rigid coupled with each other. Rotational degrees of freedom are no need to restrain.

Linear distributed temperature gradients were imposed to two planes across Y-direction which alternately cycle in phase. Two planes have same temperature range and different mean temperature which produce linearly distributed cyclic thermal bending stress and equally distributed cyclic thermal membrane stress across X- direction respectively.

Mechanical loads, e.g. force, pressure and moment, were applied to the upper boundary line of the bottom planes which cause equally distributed constant mechanical membrane stress or linearly distributed constant bending membrane stress. Note that mechanical loads are beard by two planes together because of the coupling condition.

This model have two differences compared with other similar models: 
(1)Restrictions of primary-load-bearing boundary were loosen by removing parallel condition, only rigid condition retained. The rotation of load-bearing boundary to fix boundary is allowable.

(2)Any combination of constant mechanical membrane stress and mechanical bending stress, cyclic thermal membrane stress and thermal bending stress can be considered in this ingenious model. Excessive restrictions in past models were overcome and mechanical bending stress can be easily deal with which was ignored in Bree-like problem before.

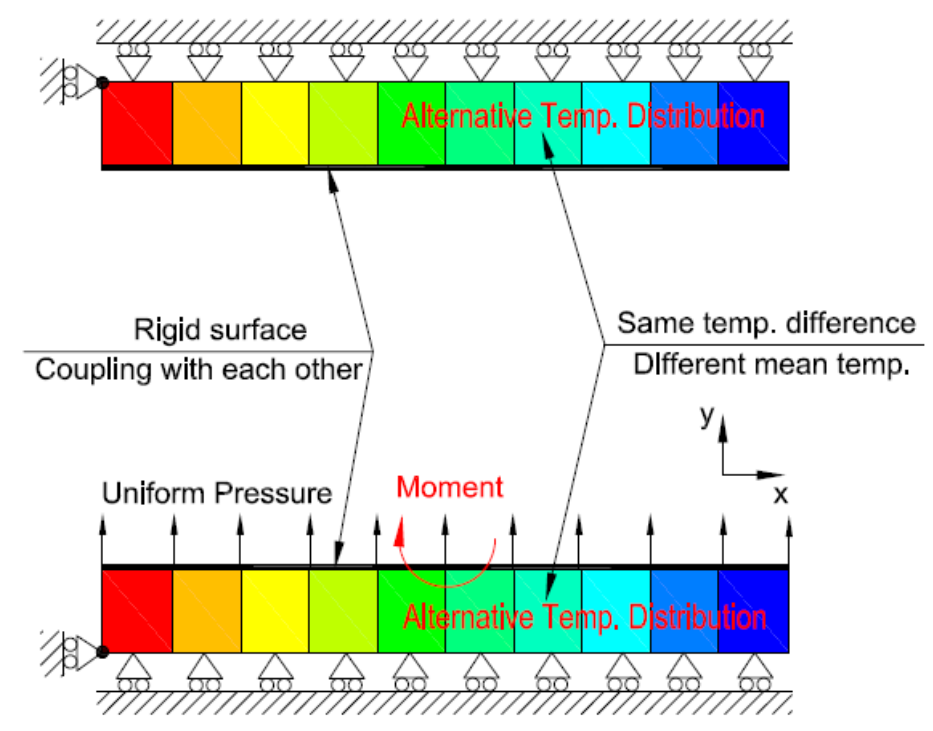

Figure 11. Two-plane FE model for validation

\subsection{Assessment criterion of ratcheting}

On ratcheting check using plastic FEA, the basic problem is the ratcheting evaluation criterion. In this paper, two practical criteria as follow are adopted.

One criterion is "elastic core criterion". Kalnins [15] proposed the use of the elastic core as a ratcheting measure for any geometry and loading. The motivation for it was that the answer to the ratcheting check could be obtained from postprocessor plots.

The second approach developed under the direction of the TDF Committee of the Japan Pressure Vessel Council (JPVRC) and summarized by Okamoto et al [16] uses the ratcheting measure of equivalent plastic strain. The ratcheting check passes if the equivalent plastic strain increments at all plastically cycled points in the model exhibit a decreasing trend with cycles and the maximum value of the increments is less than 0.0001 . The number of cycles to reach this value is not specified but 50 cycles in the analysis of this paper.

For Bree's solution he core size remains constant after the first cycle while in the other cases, e.g. transient thermal example, it may not. However, Bree's solution is only the special case of the solution of this paper. So, in the validation of this paper, these two criteria must be simultaneously satisfied

\subsection{Analysis using ANSYS}

ANSYS Workbench is used for the analysis and calculation. The dimensions of geometry see Figure 12. 


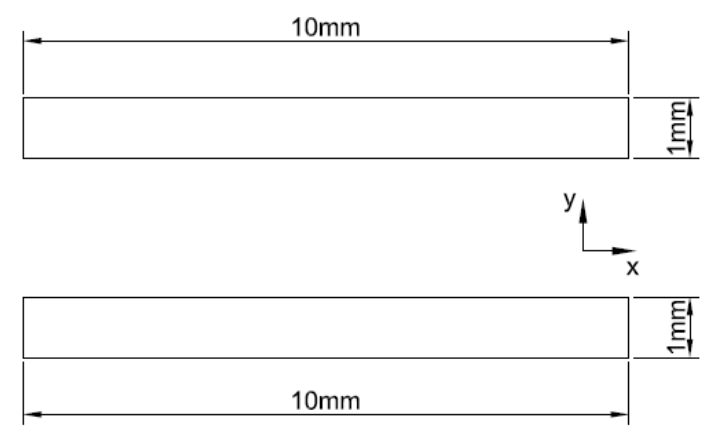

Figure 12. Dimensions of geometry

Material property is shown in Table 1 . An elastic perfectly plastic material model with small displacement theory shall be used in the analysis. The von-Mises yield function and associated flow rule should be utilized. Von-Mises yield condition is identical to Tresca yield condition in a unidirectional stress state.

Table 1. Material property

\begin{tabular}{|l|c|}
\hline Item & $\begin{array}{c}\text { Valu } \\
\mathrm{e}\end{array}$ \\
\hline Young's modulus [MPa] & $2 \mathrm{E} 5$ \\
\hline Poisson's ratio & 0.3 \\
\hline Yield strength [MPa] & 300 \\
\hline $\begin{array}{l}\text { Coefficient of thermal expansion } \\
\left.{ }^{\circ} \mathrm{C}^{-1}\right]\end{array}$ & $1 \mathrm{E}-5$ \\
\hline
\end{tabular}

The meshing is shown in the Figure 13. There are 10 elements across X-direction and two integration points per element, giving the total of 20 output points. And there are 3 elements across Y-diretion.PLANE183, which is a higher order 2-D, 8-node element, is adopted for plane stress analysis.

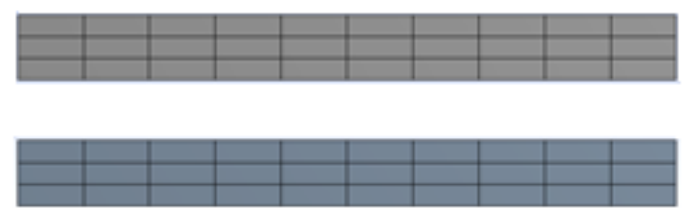

Figure 13. Meshing

Load and displacement boundary conditions are applied as per what is shown in Figure 11. And then constant mechanical membrane and bending stresses and cyclic thermal mechanical membrane and bending stresses are available. The number of cycles for thermal stress is 50 .

Next, detail procedures for determination of ratcheting boundary is provided by taking $\sigma_{\mathrm{pb}} / \sigma_{\mathrm{s}}=0.3, \sigma_{\mathrm{sb}} / \sigma_{\mathrm{s}}=1.2$ and $\sigma_{\mathrm{sm}} / \sigma_{\mathrm{s}}=0.6$ as an example. When various values of $\sigma_{\mathrm{pm}} / \sigma_{\mathrm{s}}$ are taken for calculation, a series of strain increments are available. Under the precondition of ensuring the existing of elastic core (i.e. the first ratcheting evaluation criterion), the strain 
increments of the last four cycle examined according to the second ratcheting evaluation criterion. In this illustrative example, some of the key strain increments data is shown in Table 2. As we can see, variations in equivalent plastic strain increments at the end of the last four cycles have a decreasing trend in all three columns and become lower than the allowable limit $10 \mathrm{E}-4$ in the first and second columns. Obviously, the data in second column is much closer to allowable limit than that in first column. So FEA result of $\sigma_{\mathrm{pm}} / \sigma_{\mathrm{s}}$ for the corresponding ratcheting boundary in this example is identified as 0.3353 whose relative deviation is $0.98 \%$ to the analytic solution 0.33206 . The FEA results agree with the theory perfectly.

Table 2. Strain increments per cycle

\begin{tabular}{|c|c|c|c|}
\hline $\begin{array}{c}\text { Cycle } \\
\text { No. }\end{array}$ & $\begin{array}{c}\sigma_{\mathrm{pm}} / \sigma_{\mathrm{s}}= \\
0.3320\end{array}$ & $\begin{array}{c}\sigma_{\mathrm{pm}} / \sigma_{\mathrm{s}}= \\
0.3353\end{array}$ & $\begin{array}{c}\sigma_{\mathrm{pm}} / \sigma_{\mathrm{s}}= \\
0.3369\end{array}$ \\
\hline 47 & 0.000081 & 0.000097 & $\begin{array}{c}0.00010 \\
5\end{array}$ \\
\hline 47 & 0.000080 & 0.000095 & $\begin{array}{c}0.00010 \\
3\end{array}$ \\
\hline 49 & 0.000079 & 0.000094 & $\begin{array}{c}0.00010 \\
2\end{array}$ \\
\hline 50 & 0.000078 & 0.000093 & $\begin{array}{c}0.00010 \\
2\end{array}$ \\
\hline
\end{tabular}

\subsection{Results of validation}

The ratcheting boundary of any load point consisted in any reasonable combination of $\sigma_{\mathrm{pm}} / \sigma_{\mathrm{s}}, \sigma_{\mathrm{pb}} / \sigma_{\mathrm{s}}, \sigma_{\mathrm{sm}} / \sigma_{\mathrm{s}}$ and $\sigma_{\mathrm{sb}} / \sigma_{\mathrm{s}}$ is provided in the above section. Some decomposition of the four-dimensional ratcheting boundary analytic solution is essential before its full validation. The methods of decomposition is as follows. Any one of the four involved stress parameters should be set to a series of fixed values to obtain a series of three-dimensional solutions corresponding to each fixed value. Here $\sigma_{\mathrm{pb}} / \sigma_{\mathrm{s}}$ is selected as such fixed parameter so that Bree-style diagram, that is, abscissa and ordinate are $\sigma_{\mathrm{pm}} / \sigma_{\mathrm{s}}$ and $\sigma_{\mathrm{sb}} / \sigma_{\mathrm{s}}$, will obtained in final validation diagrams. The purpose of doing so is for consistency with predecessors and easy understanding.

More specifically, $\sigma_{\mathrm{pb}} / \sigma_{\mathrm{s}}$ is set to $0,0.3,0.6,0.9,1.2,1.5$ respectively. Six corresponding three- dimensional solutions are determined and their three-dimensional curved surface are shown in Figure 14-19. There is only a point in Figure 19 representing plastic limit bending moment under pure bending condition.

For the first five three- dimensional solutions, $\sigma_{\mathrm{sm}} / \sigma_{\mathrm{s}}$ is set to $0,0.5,1.0,1.5$ and 2.0 respectively. Then five contour plots with contour lines are obtained for each $\sigma_{\mathrm{pb}} / \sigma_{\mathrm{s}}$ shown in Figure 14-18 in three-dimensions and Figure 20-24 in two-dimensions by projection. Especially, the intersection of ratcheting boundary surface in Figure 14 and the left coordinate 
plane and the rightmost black line in Figure 20 is the distinguished Bree's solution.

For each contour line, $\sigma_{\mathrm{sb}} / \sigma_{\mathrm{s}}$ is set to $0,1,2,3,4$ and5 respectively to determine the limit value of $\sigma_{\mathrm{pm}} / \sigma_{\mathrm{s}}$ as per the two criteria above. The point determined by $\sigma_{\mathrm{pm}} / \sigma_{\mathrm{s}}$ and $\sigma_{\mathrm{sb}} / \sigma_{\mathrm{s}}$ will be superimposed into contour plots to verify analytic solutions straightforward. Note that, not all six values of $\sigma_{\mathrm{sb}} / \sigma_{\mathrm{s}}$ will be used for each contour line.

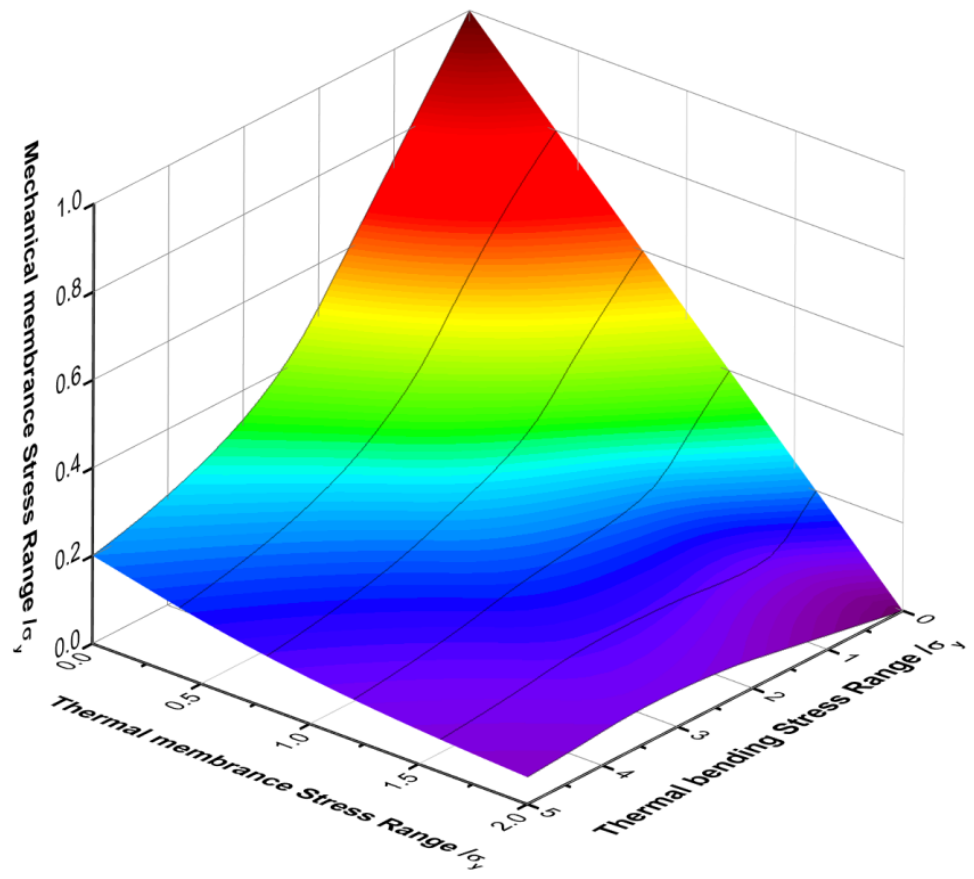

Figure 14. Ratchet boundary with contour lines of thermal membrane stress when mechanical bending stress $/ \sigma_{\mathrm{y}}=0$ 


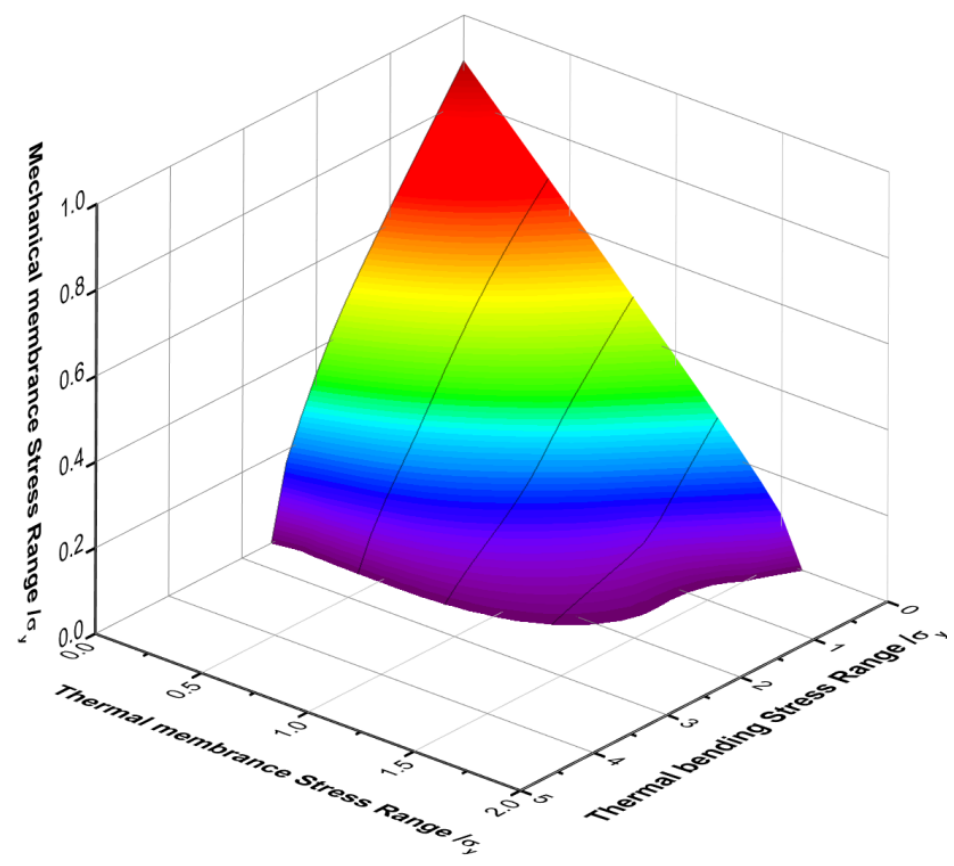

Figure 15. Ratchet boundary with contour lines of thermal membrane stress when mechanical bending stress $/ \sigma_{\mathrm{y}}=0.3$

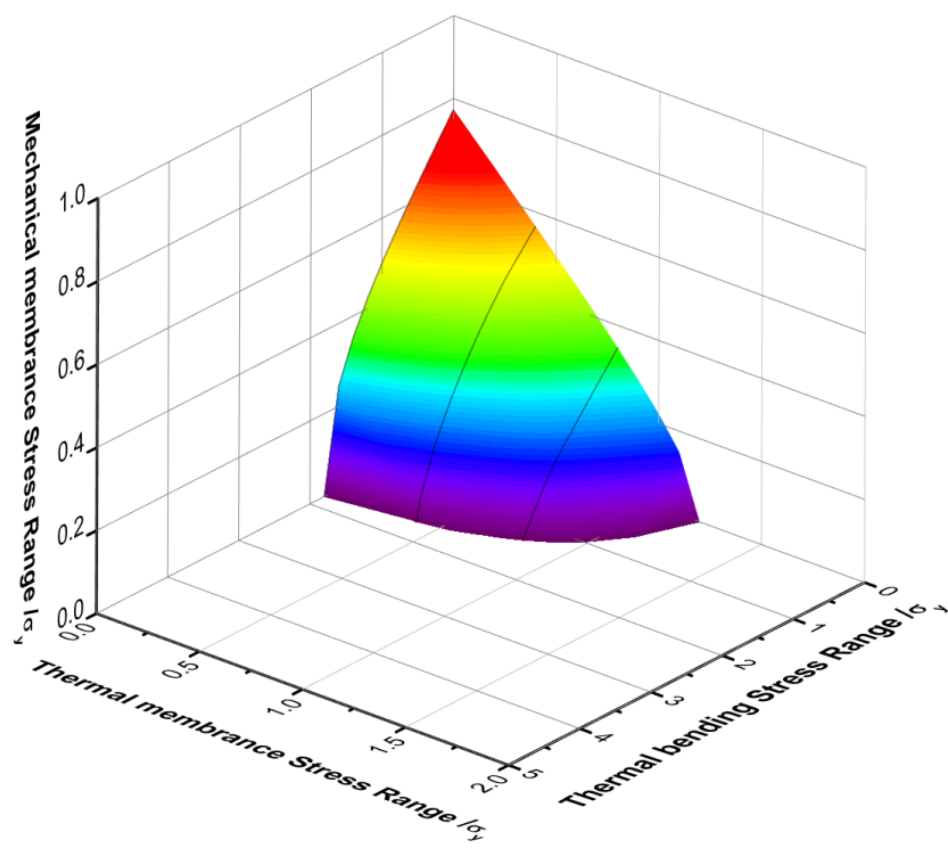

Figure 16. Ratchet boundary with contour lines of thermal membrane stress when mechanical bending stress $/ \sigma_{\mathrm{y}}=0.6$ 


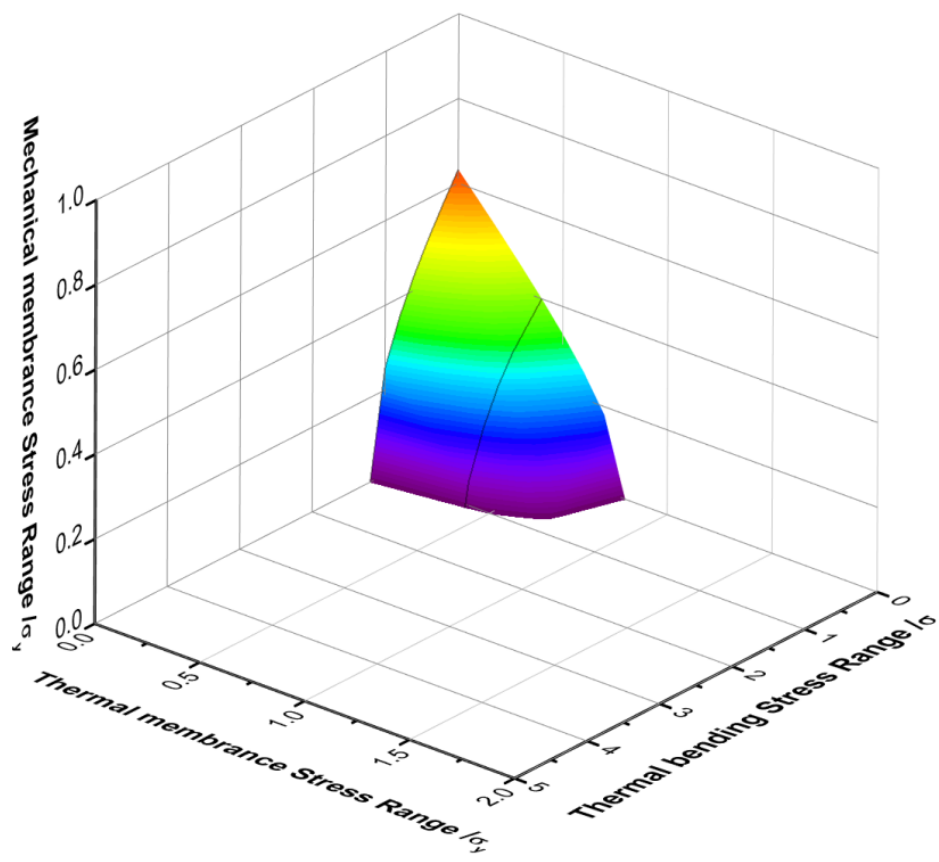

Figure 17. Ratchet boundary with contour lines of thermal membrane stress when mechanical bending stress $/ \sigma_{\mathrm{y}}=0.9$

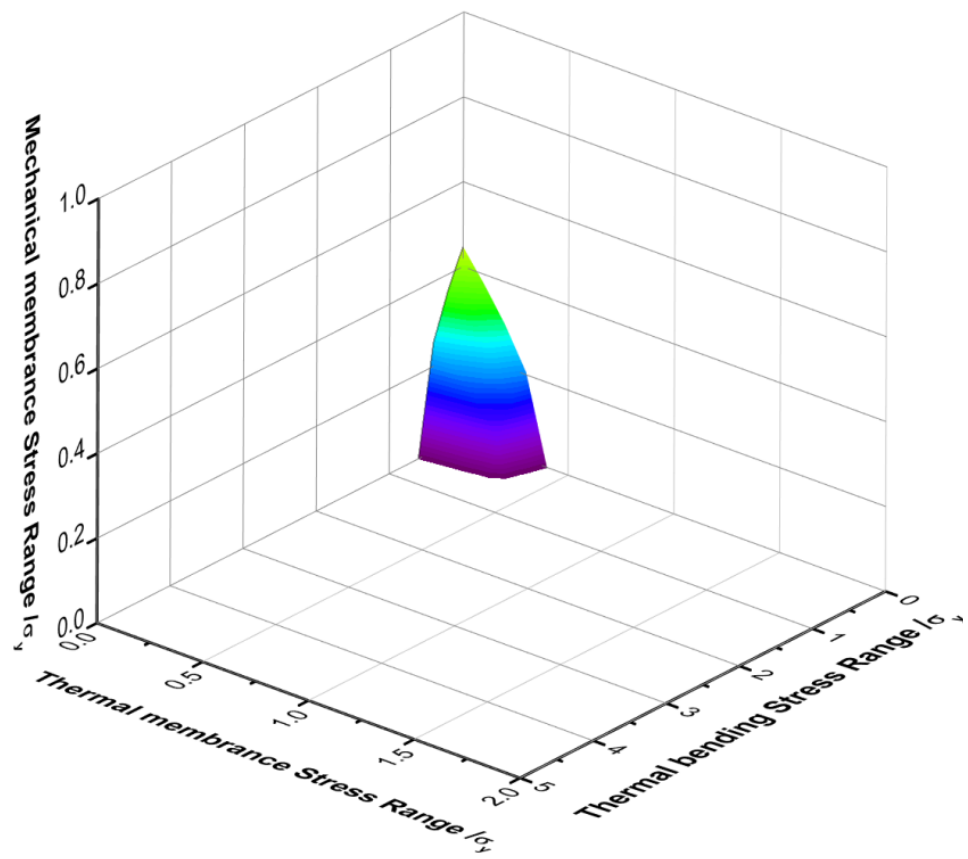

Figure 18. Ratchet boundary with contour lines of thermal membrane stress when mechanical bending stress $/ \sigma_{\mathrm{y}}=1.2$ 


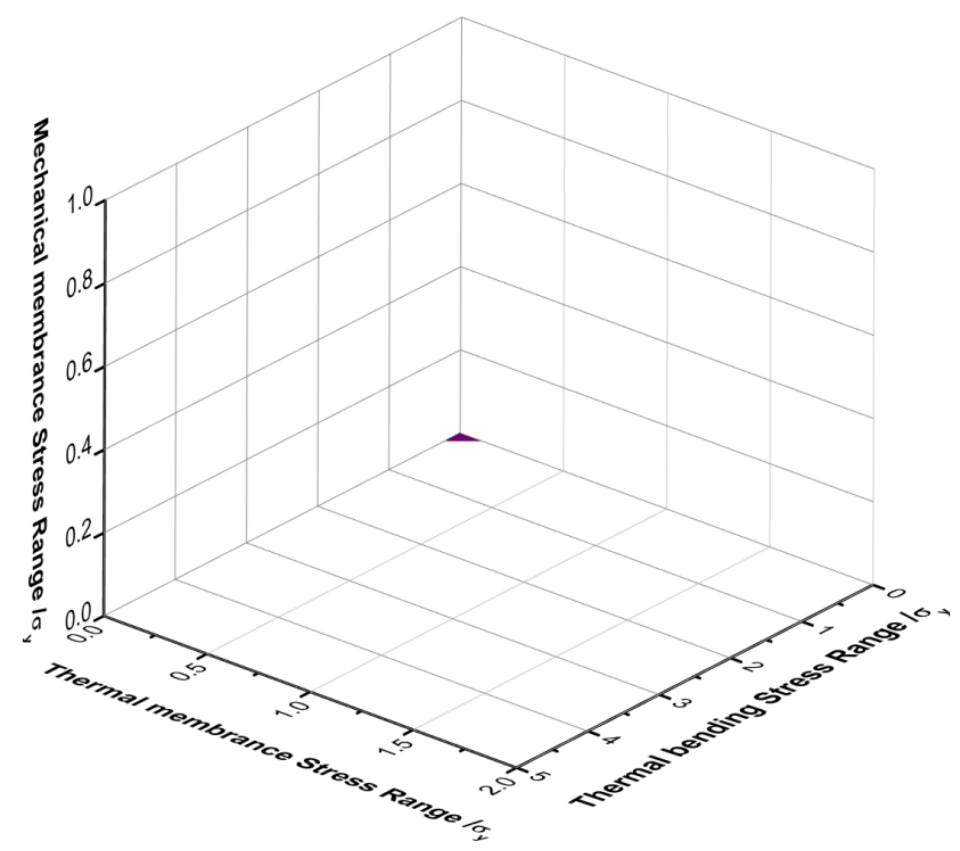

Figure 19. Ratchet boundary with contour lines of thermal membrane stress when mechanical bending stress $/ \sigma_{\mathrm{y}}=1.5$

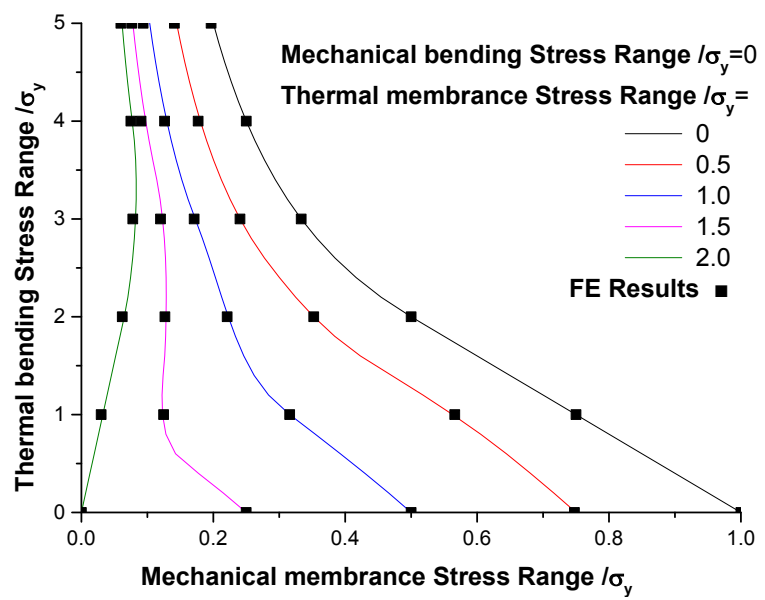

Figure 20. Projection of contour lines of different $\sigma_{\mathrm{sm}} / \sigma_{\mathrm{s}}$ in Figure 14 with FE result

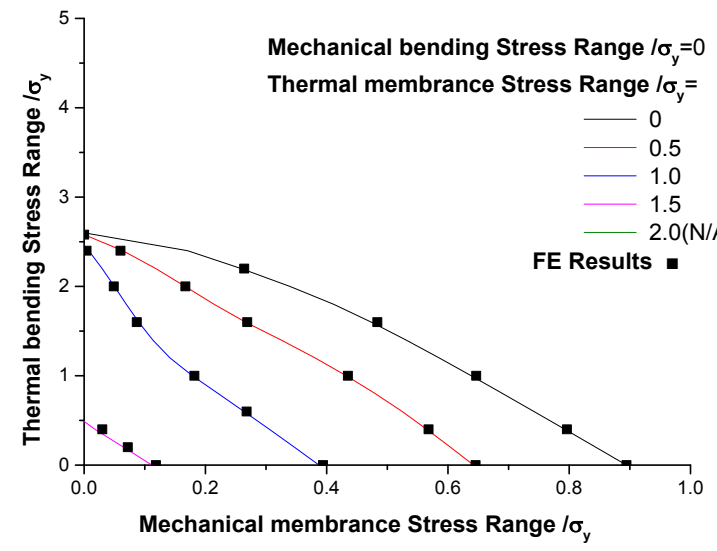


Figure 21. Projection of contour lines of different $\sigma_{\mathrm{sm}} / \sigma_{\mathrm{s}}$ in Figure 15 with FE result

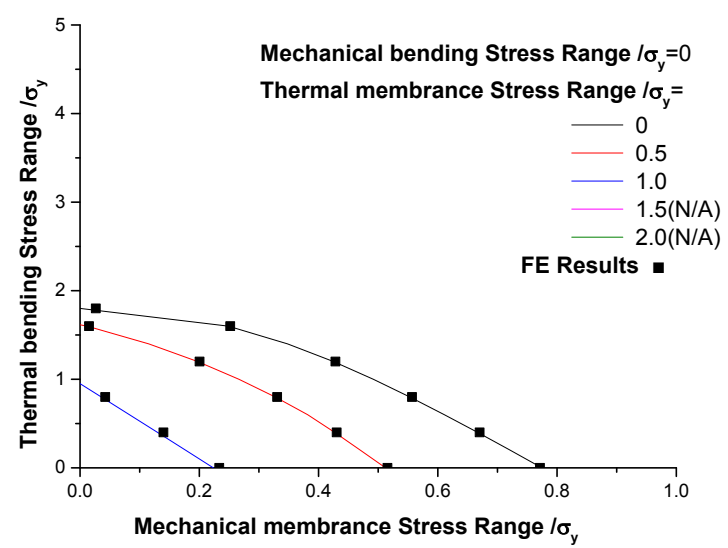

Figure 22. Projection of contour lines of different $\sigma_{\mathrm{sm}} / \sigma_{\mathrm{s}}$ in Figure 16 with FE result

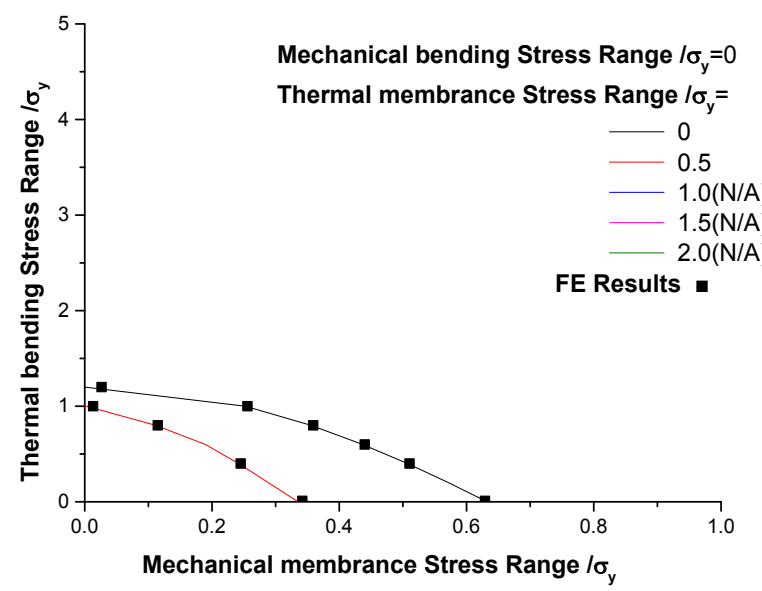

Figure 23. Projection of contour lines of different $\sigma_{\mathrm{sm}} / \sigma_{\mathrm{s}}$ in Figure 17 with FE result

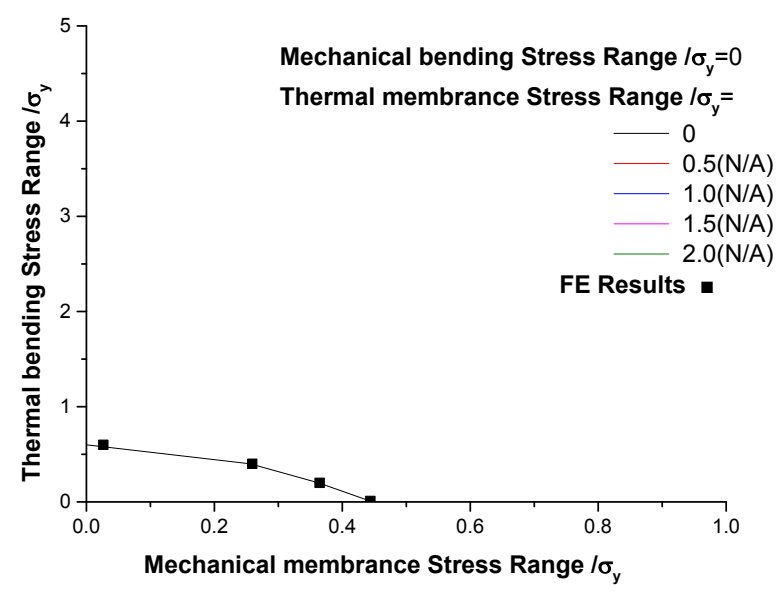


Figure 24. Projection of contour lines of different $\sigma_{\mathrm{sm}} / \sigma_{\mathrm{s}}$ in Figure 18 with FE result

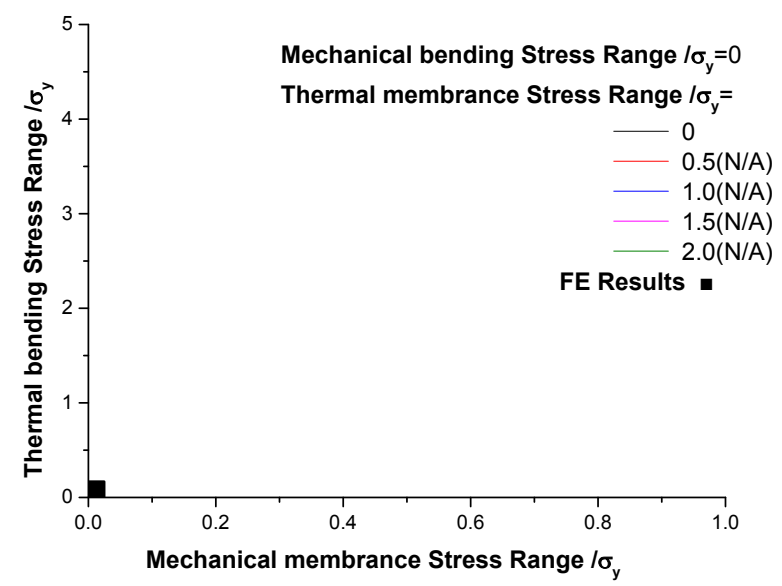

Figure 25. Projection of contour lines of different $\sigma_{\mathrm{sm}} / \sigma_{\mathrm{s}}$ in Figure 19 with FE result

By calculating 116 four-dimensional load points representing a combination of four stresses, current unified four-dimensional analytical solution for ratcheting boundary has been verified completely.

\section{Conclusions}

A new four-dimensional ratchet boundary considering constant mechanical membrane stress and mechanical bending stress, cyclic thermal membrane stress and thermal bending stress simultaneously were investigated and validated systematically. The main conclusions include:

(1) Compared to the previous researches, the analytical solution gives out unified ratcheting boundary for any combination of four types of stress and can also be easily degenerated to the Bree problem considering the constant mechanical membrane stress and cyclic thermal bending stress, inverse Bree problem in view of the mechanical bending stress and thermal membrane stress, and the solution considering thermal bending stress, mechanical membrane stress and mechanical bending stress.

(2) The derived solution as the mechanical bending stress reduces to zero is more conservative to the solution which however ignored the equilibrium equation of mechanical bending moment. When thermal bending and membrane stresses simultaneously exist, the influence of mechanical bending stress cannot be ignored, unless the primary-load-bearing boundary is forced to keep parallel to fixed displacement bounding that is not a general case, but a special case in engineering. Otherwise, non-conservative and unsafety result may occur.

(3) Some interesting and practical results are drawn that for some certain combinations of thermal bending stress and thermal membrane stress, the mechanical bending stress may promote the loading capacity of structure for mechanical membrane stress and vice versa. However, if the thermal stress only includes the thermal bending stress or the thermal membrane stress, the increment of the mechanical membrane stress will shrink the region of shakedown for mechanical bending stress and vice versa. All above conclusions can guide the design of actual structure.

(4) The non-cyclic methods are used to determine the ratcheting boundary of structural 
components. It is shown that these methods are simple, efficient and accurate for the given examples. X.H. Chen et al. [17] reached the same conclusions.

(5) A novel and flexible two-plane FE model was built for conveniently considering more kinds of stresses and load-bearing boundary than ever before. The functionality of this model can also be extended for other type of cross-validation (e.g., biomaterial problem).

(6) There are not many analytical solutions of ratchetting boundary, especially complicated stress conditions. The result in the present paper is beneficial and can be used as references, benchmarks or cross validations for the research in experimental investigation and finite element analysis of ratcheting.

\section{Acknowledgments}

The authors are gratefully acknowledge the support of the National Science Foundation for Distinguished Young Scholars of China (Grant No.11325211) and the Project of International Cooperation and Exchange NSFC (Grant No. 11511130057) during this work.

\section{References}

[1] X.T. Zheng, HY Peng, JY Yu, etc. Analytical Ratchet Limit for Pressurized Pipeline under Cyclic Nonproportional Loadings. Pipeline Syst. Eng. Pract.2017; 8: 04017002.

[2] R.A.W. Bradford, D.J. Tipping, The Ratchet-Shakedown Diagram for a Thin Pressurized Pipe Subject to Additional Axial Load and Cyclic Secondary Global Bending, Press Vessel Technol-Trans ASME. 2015; 92-100.

[3] J. Bree. Elastic-Plastic Behaviour of Thin Tubes Subjected to Internal Pressure and Intermittent High Heat Fluxes with Application to Fast Nuclear Reactor Fuel Elements. Strain Anal. 1967;2: 226-238.

[4] J. Bree, "Plastic Yielding of Thin Tubes Subjected to Internal Pressure and Cyclic Thermal Stresses", Journal of Strain Analysis (1968) 3, 122-127.

[5] ASME Boiler \& Pressure Vessel Code. Alternative Rules for Construction of Pressure Vessels. VIII Division 2, 2011.

[6]Bradford, R. A. W. "The Bree problem with primary load cycling in-phase with the secondary load." International Journal of Pressure Vessels \& Piping 99-100.5(2012):44-50.

[7]Bradford, R. A. W., J. Ure, and H. F. Chen. "The Bree problem with different yield stresses on-load and offload and application to creep ratcheting." International Journal of Pressure Vessels \& Piping 113.1(2014):32-39.

[8] Reinhardt W. On the interaction of thermal membrane and thermal bending stress in shakedown analysis. New York: American Society Mechanical Engineers. 2009.

[9] Adibi-Asl R, Reinhardt W. Ratchet Limit Solution of a Beam With Arbitrary Cross Section. Press Vessel Technol-Trans ASME. 2015;137.

[10] H.F.Chen and A.R.S.Ponter, "Linear Matching Method on the Evaluation of Plastic and Creep Behaviours for Bodies Subjected to Cyclic Thermal

[11] H.F.Chen, "Lower and upper bound shakedown analysis of structures with temperature dependent yield stress", Journal of Pressure Vessel Technology 2010;132(1) p.011202: 1-8.

[12] ASME Boiler \& Pressure Vessel Code. Alternative Rules for Construction of Pressure Vessels. VIII Division $2,2013$.

[13] Reinhardt W. A noncyclic method for plastic shakedown analysis. Press Vessel Technol-Trans ASME. 2008;130:6.

[14] Yukinori Yamamoto, Norimichi Yamashita and Masaaki Tanaka, Evaluation of Thermal Stress Ratchet in Plastic FEA ASME PVP2002-1215, pp. 3-10; 8 pages

[15]. Kalnins, A, Shakedown check for pressure vessel using plastic FEA. ASME PVP2001; 419; 9-16

[16] Okamoto A, Nishiguchi I and Aoki M. Recent Advancement on the Draft of Alternate Stress Evaluation Criteria in Japan Based on Partial Inelastic Analyses. ASME PVP 2010; 419: 17-24.

[17]Chen, X., et al. "Recent progresses in experimental investigation and finite element analysis of ratcheting in pressurized piping." International Journal of Pressure Vessels \& Piping 101.101(2013):113-142. 